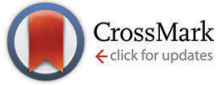

Cite this: Phys. Chem. Chem. Phys., 2016, 18, 18470

Received 24th May 2016, Accepted 15th June 2016

DOI: $10.1039 / c 6 c p 03555 h$

www.rsc.org/pccp

\title{
Coherent pump pulses in Double Electron Electron Resonance spectroscopy $\dagger$
}

\begin{abstract}
Claudia E. Tait and Stefan Stoll*
The recent introduction of shaped pulses to Double Electron Electron Resonance (DEER) spectroscopy has led to significant enhancements in sensitivity through increased excitation bandwidths and improved control over spin dynamics. The application of DEER has so far relied on the presence of an incoherent pump channel to average out most undesired coherent effects of the pump pulse(s) on the observer spins. However, in fully coherent EPR spectrometers that are increasingly used to generate shaped pulses, the presence of coherent pump pulses means that these effects need to be explicitly considered. In this paper, we examine the effects of coherent rectangular and sech/tanh pump pulses in DEER experiments with up to three pump pulses. We show that, even in the absence of significant overlap of the observer and pump pulse excitation bandwidths, coherence transfer pathways involving both types of pulses generate spin echoes of considerable intensity. These echoes introduce artefacts, which, if not identified and removed, can easily lead to misinterpretation. We demonstrate that the observed echoes can be quantitatively modelled using a simple spin quantum dynamics approach that includes instrumental transfer functions. Based on an analysis of the echo crossing artefacts, we propose efficient phase cycling schemes for their suppression. This enables the use of advanced DEER experiments, characterized by high sensitivity and increased accuracy for long-distance measurements, on novel fully coherent EPR spectrometers.
\end{abstract}

\section{Introduction}

Double Electron Electron Resonance (DEER), or Pulsed ELectron DOuble Resonance (PELDOR), experiments are used in Electron Paramagnetic Resonance (EPR) spectroscopy to determine the structure and conformational dynamics of macromolecular systems by measuring the distance between intrinsic or artificially introduced paramagnetic centres through their dipolar interaction., ${ }^{1,2}$ This technique is most widely applied to investigate proteins and protein complexes labelled at different positions with nitroxide spin labels introduced by site-directed mutagenesis. ${ }^{3-7}$

DEER is a multi-frequency experiment for which the most commonly used pulse sequence is the four-pulse sequence depicted in Fig. 1. The intensity of the echo at the end of a refocused primary echo sequence at an observer frequency is monitored as a function of the position of a $\pi$ pulse at a second microwave frequency (pump frequency). The pump pulse causes inversion of the pump spins (B spins) coupled to the observer spins (A spins), which induces a change in the local field of

Department of Chemistry, University of Washington, Seattle, WA 98195, USA. E-mail: stst@uw.edu

$\dagger$ Electronic supplementary information (ESI) available: Additional experimental details and optimization procedures, additional simulations, lists of echoes generated by five-pulse DEER sequences and details on the phase cycles. See DOI: $10.1039 / \mathrm{c} 6 \mathrm{cp} 03555 \mathrm{~h}$ the A spins. As a result, the echo is modulated at the frequency of the dipolar coupling between the two spins, and the distance between them can be extracted..$^{8,9}$ Ideally, the pump pulse does not directly affect the observer spins; it has however been shown that this is not the case experimentally. Off-resonant effects lead to dynamic phase shifts in the refocused echo, ${ }^{10}$ and overlap of the excitation profiles of observer and pump pulses can induce nuclear modulation or ' $2+1$ '-pulse-traintype dipolar modulation artefacts. ${ }^{11,12}$ Additional instrumental artefacts can also appear when pump and observer pulses are separated by short time delays.

A high level of sensitivity is required for the reliable extraction of accurate distance information. The sensitivity of fourpulse DEER experiments is limited on one hand by the intensity of the detected observer echo, depending on the phase memory time and the overall length of the DEER sequence, and on the other by the modulation depth, depending on the number of spins inverted by the pump pulse. The length of the DEER sequence determines the range of inter-spin distances that can be reliably detected. ${ }^{12}$

The introduction of shaped pulses to EPR, enabled by the availability of arbitrary waveform generators (AWGs) with nanosecond resolution, has provided new possibilities for the improvement of current pulse EPR experiments, including DEER. ${ }^{13-23}$ The increased excitation bandwidth of linear chirp 
Four-pulse DEER

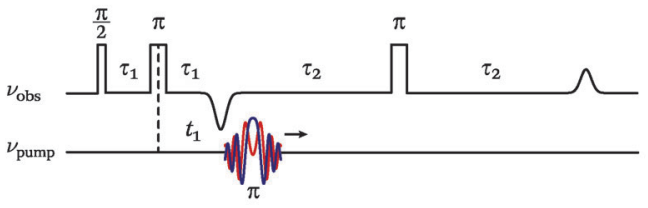

Forward five-pulse DEER

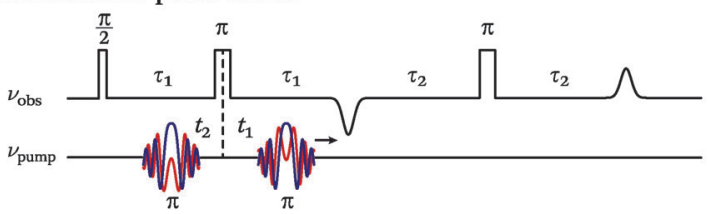

Reverse five-pulse DEER

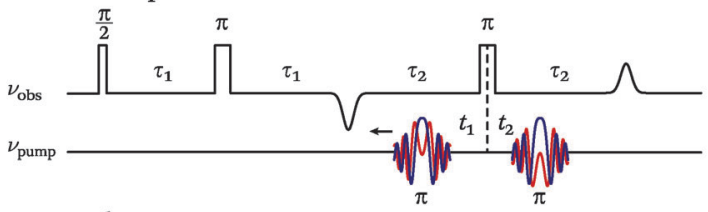

Seven-pulse DEER

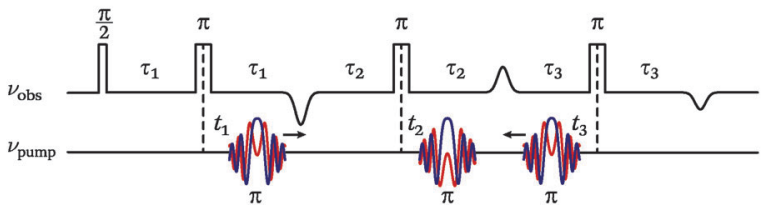

Fig. 1 Schematic representation of the pulse sequences for four-pulse, the forward and reverse versions of five-pulse, and seven-pulse DEER. The time axis of the DEER experiment is obtained as $t_{D E E R}=t_{1}-\tau_{1}$ for the fourpulse experiment, $t_{\mathrm{DEER}}=t_{1}-t_{2}$ for the five-pulse DEER experiments and $t_{\text {DEER }}=\tau_{1}+\tau_{2}+\tau_{3}-t_{1}-t_{2}-t_{3}$ for the seven-pulse DEER experiment.

or hyperbolic secant/hyperbolic tangent (sech/tanh) pump pulses has been exploited to increase the modulation depth and therefore the sensitivity of the DEER experiment. ${ }^{15,16,20}$ One further advance in this technique is the extension of the maximum length of the DEER traces through modification of the pulse sequence, with the aim to provide access to longer inter-spin distances. A five-pulse DEER experiment (see Fig. 1) has been proposed, where similar lengths of the inter-pulse delays $\tau_{1}$ and $\tau_{2}$ reduce the effect of nuclear spin diffusion on relaxation, achieving higher observer echo intensities, and an additional pump pulse has been included to extend the dipolar evolution window. ${ }^{14,16}$ A seven-pulse Carr-Purcell PELDOR experiment with multiple pump pulses has also been proposed (see Fig. 1) and allows for an even more substantial increase in the length of the dipolar evolution time with comparable sensitivity. ${ }^{21}$

The AWG capabilities required for the use of shaped pump pulses have initially been introduced as home-built extensions to existing EPR spectrometers. ${ }^{13-15}$ AWG-based spectrometers have also been developed ${ }^{17,24}$ and have recently become commercially available.

The application of shaped pump pulses in DEER has mainly been limited to instruments equipped with two separate incoherent microwave sources for the observer and pump pulses, i.e. with an incoherent pump channel. Several developed home-built and the commercially available AWG-based spectrometers however use a single microwave source and therefore generate coherent observer and pump pulses. While in the presence of an incoherent pump channel most effects of the pump pulse on the observer A spins are averaged out by the random phase of the pump pulse with respect to the observer pulses, this is no longer true for a coherent spectrometer. Coherence transfer pathways involving the pump pulse can generate echoes that interfere with the desired signal and therefore introduce artefacts in DEER traces recorded with a coherent pump pulse. These artefacts, if they remain unidentified and are interpreted as part of the dipolar oscillations, can lead to the extraction of erroneous distance distributions and misinterpretation of the experimental data. A detailed understanding of the origin and nature of these artefacts is therefore required to devise strategies for their removal.

In this paper, we investigate the effects of coherent pulses in multi-frequency experiments on the example of the four-, five-, and seven-pulse DEER experiments on nitroxide-labelled proteins. We present a detailed analysis of the consequences of coherent pump and observer frequencies under a range of experimental conditions.

We analyse the shapes and relative intensities of the different types of interfering echoes generated in experiments with coherent pump pulses and demonstrate that they can be modelled quantitatively using a simple density matrix approach that takes the instrumental transfer functions into account.

Based on the results of the analysis of these additional echo signals, the different factors influencing the severity of the distortions introduced in DEER are discussed. It is shown that elimination of the introduced echo-crossing artefacts can be achieved by phase cycling. Efficient phase cycling schemes for the three types of DEER experiments are proposed, enabling the practical use of these advanced DEER sequences with increased sensitivity on novel coherent AWG-based spectrometers.

\section{Four-pulse DEER}

\subsection{Coherence transfer pathways and echoes}

DEER traces recorded with the four-pulse sequence using a monochromatic pump pulse on an incoherent or coherent channel at the pump frequency and no phase cycling are compared in Fig. 2. The use of coherent pump pulses leads to significant distortions at several points of the DEER trace. Inspection of the transient signals for different positions of the pump pulse (see Fig. 2) reveals the presence of multiple moving echoes in addition to the standing echoes generated by the three observer pulses. These additional echoes are generated by coherence transfer pathways $^{25-27}$ involving both observer and pump pulses and cross the detected refocused primary echo $\left(\mathrm{PE}_{12} \mathrm{r}_{3}\right.$ at $2 \tau_{1}+2 \tau_{2}$, see Table 1 for echo notation) at different times in the DEER experiment, leading to the observed artefacts. Similar artefacts are also observed for coherent sech/tanh pump pulses (discussed below). Phase shifts and an apparent oscillation are present at the end of the DEER trace for both experiments with coherent and incoherent pump pulses. These features are independent of sample and measurement conditions and are likely instrumental 

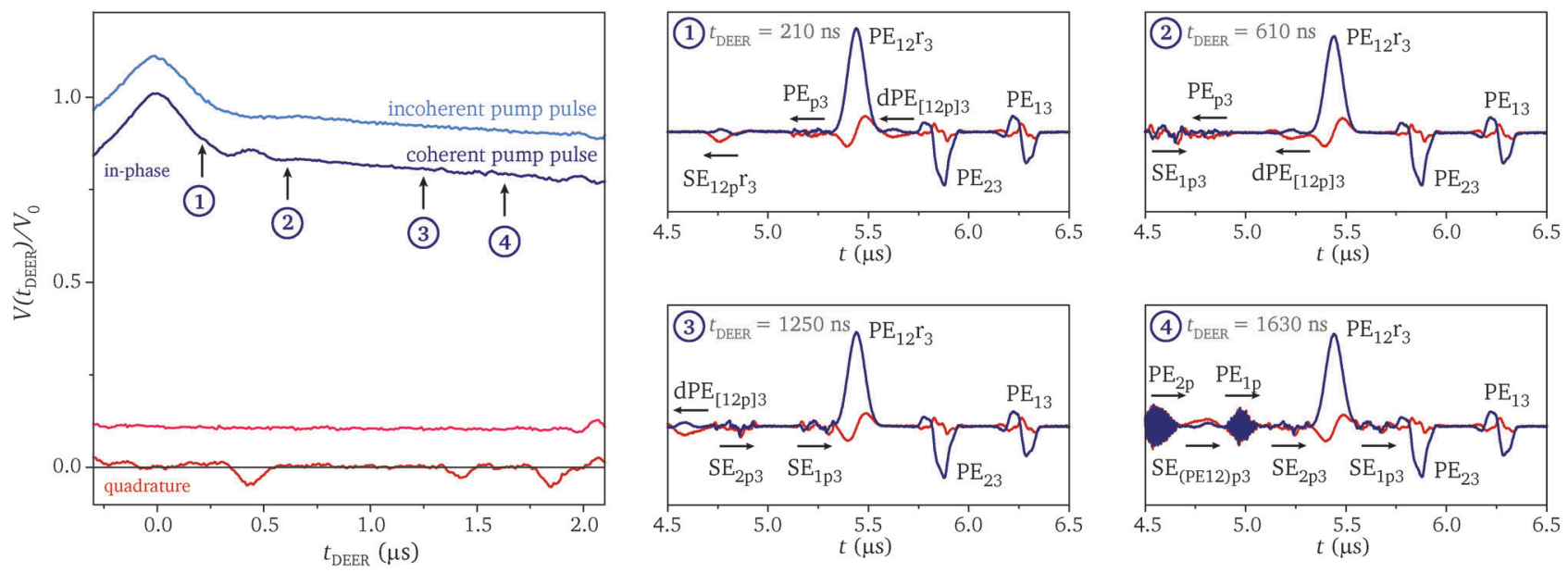

Fig. 2 Comparison of the in-phase (top) and quadrature (bottom) components of four-pulse DEER traces recorded with incoherent or coherent 64 ns pump pulses at a frequency offset of $\Delta \nu=70 \mathrm{MHz}$ without phase cycling $\left(\nu_{\mathrm{obs}}=33.83 \mathrm{GHz}, B_{0}=1207.9 \mathrm{mT}, \tau_{1}=400 \mathrm{~ns}, \tau_{2}=2.3 \mu \mathrm{s}\right.$, further details in Section 5). The trace recorded with incoherent pump pulses is offset for better comparison. The echo transients recorded for four positions of the coherent pump pulse are shown on the right. The in-phase and quadrature components are shown in blue and red, respectively. The different echoes are labelled according to Table 1 . The direction of movement for crossing echoes as $t_{\mathrm{DEER}}$ is increased is indicated by arrows.

Table 1 Echoes, coherence transfer pathways, positions and crossing times $t_{\text {DEER,cross }}$ with the refocused echo $\left(\mathrm{PE}_{12} r_{3}\right)$ for a four-pulse DEER sequence $\left(t_{\mathrm{DEER}}=t_{1}-\tau_{1}\right)$. The coherence transfer pathways are specified by denoting the observer coherence order $(-1,0,+1)$ during each of the delays of the pulse sequence. $(\mathrm{PE}=$ primary echo, $\mathrm{SE}=$ stimulated echo, $r=$ refocused, $d=$ delayed.)

\begin{tabular}{|c|c|c|c|c|c|c|}
\hline \multirow{2}{*}{$\frac{\text { Echo }}{\mathrm{PE}_{12}}$} & \multicolumn{4}{|c|}{ Pathway } & \multirow{2}{*}{$\frac{\text { Position }}{2 \tau_{1}}$} & \multirow{2}{*}{$\frac{t_{\mathrm{DEER}, \mathrm{cross}}}{-}$} \\
\hline & + & - & - & - & & \\
\hline $\mathrm{PE}_{1 \mathrm{p}}$ & + & + & - & - & $2 \tau_{1}+2 t_{1}$ & $\tau_{2}-\tau_{1}$ \\
\hline $\mathrm{PE}_{2 p}$ & 0 & + & - & - & $\tau_{1}+2 t_{1}$ & $\tau_{2}-\frac{1}{2} \tau_{1}$ \\
\hline $\mathrm{PE}_{13}$ & + & + & + & - & $4 \tau_{1}+2 \tau_{2}$ & - \\
\hline $\mathrm{PE}_{23}$ & 0 & + & + & - & $3 \tau_{1}+2 \tau_{2}$ & - \\
\hline $\mathrm{PE}_{\mathrm{p} 3}$ & 0 & 0 & + & - & $3 \tau_{1}+2 \tau_{2}-t_{1}$ & 0 \\
\hline $\mathrm{PE}_{12} \mathrm{r}_{\mathrm{p}}$ & - & + & - & - & $2 t_{1}$ & $\tau_{2}$ \\
\hline $\mathbf{P E}_{12} \mathbf{r}_{3}$ & - & + & + & - & $2 \tau_{1}+2 \tau_{2}$ & Detection \\
\hline $\mathrm{PE}_{1 \mathrm{p}} \mathrm{r}_{3}$ & - & - & + & - & $2 \tau_{1}+2 \tau_{2}-2 t_{1}$ & $-\tau_{1}$ \\
\hline $\mathrm{PE}_{2 \mathrm{p}} \mathrm{r}_{3}$ & 0 & - & + & - & $3 \tau_{1}+2 \tau_{2}-2 t_{1}$ & $-\frac{1}{2} \tau_{1}$ \\
\hline $\mathrm{PE}_{12} \mathrm{r}_{\mathrm{p}} \mathrm{r}_{3}$ & + & - & + & - & $4 \tau_{1}+2 \tau_{2}-2 t_{1}$ & $0^{21}$ \\
\hline $\mathrm{dPE}_{[12 \mathrm{p}] 3}$ & + & 0 & + & - & $4 \tau_{1}+2 \tau_{2}-t_{1}$ & $\tau_{1}$ \\
\hline $\mathrm{dPE}_{1[2 \mathrm{p} 3]}$ & + & - & 0 & - & $3 \tau_{1}+\tau_{2}-t_{1}$ & $-\tau_{2}$ \\
\hline $\mathrm{SE}_{12 \mathrm{p}}$ & + & 0 & - & - & $2 \tau_{1}+t_{1}$ & $2 \tau_{2}-\tau_{1}$ \\
\hline $\mathrm{SE}_{123}$ & + & 0 & 0 & - & $3 \tau_{1}+\tau_{2}$ & - \\
\hline $\mathrm{SE}_{1 \mathrm{p} 3}$ & + & + & 0 & - & $3 \tau_{1}+\tau_{2}+t_{1}$ & $\tau_{2}-2 \tau_{1}$ \\
\hline $\mathrm{SE}_{2 \mathrm{p} 3}$ & 0 & + & 0 & - & $2 \tau_{1}+\tau_{2}+t_{1}$ & $\tau_{2}-\tau_{1}$ \\
\hline $\mathrm{SE}_{12 \mathrm{p}} \mathrm{r}_{3}$ & - & 0 & + & - & $2 \tau_{1}+2 \tau_{2}-t_{1}$ & $-\tau_{1}$ \\
\hline $\mathrm{SE}_{(\mathrm{PE} 12) \mathrm{p} 3}$ & - & + & 0 & - & $\tau_{1}+\tau_{2}+t_{1}$ & $\tau_{2}$ \\
\hline
\end{tabular}

artefacts occurring as the time delay between the pump pulse and the last observer pulse decreases and the two pulses start to overlap in time.

Starting from thermal equilibrium (coherence order 0 ), the four pulses can lead to $3^{N-1}=27$ coherence transfer pathways ending with observer spin coherence order -1 for detection. Neglecting the pathways leading to FIDs and pathways that do not lead to refocusing to an echo leaves 19 coherence transfer pathways. The corresponding echoes and their refocusing points are listed in Table 1. The positions of the echoes during the four-pulse DEER experiment are shown in Fig. 3. The observer pulse sequence generates five standing echoes, which in most cases do not interfere with detection on the refocused echo $\mathrm{PE}_{12} \mathrm{r}_{3}$ at $2 \tau_{1}+2 \tau_{2}$. However, if $\tau_{1}$ and $\tau_{2}$ are equal (or almost equal), the stimulated echo $\mathrm{SE}_{123}$ at $3 \tau_{1}+\tau_{2}$ can overlap with the detection window and needs to be removed by phase cycling. The remaining 14 echoes cross the refocused echo at various values of the delay $t_{\mathrm{DEER}}$ (see Table 1 ). If $t_{\mathrm{DEER}}$ is varied within the range $-\tau_{1} \leq t_{\text {DEER }} \leq \tau_{2}$, distortions of the DEER trace due to crossing echoes can occur at the positions indicated on top of Fig. 3. The echo crossings at $-\tau_{1}$ and $\tau_{2}$ are generally not a concern, as this part of the trace is not recorded due to observer and pump pulse overlap.

\subsection{Echoes generated by coherent pump pulses}

The transients in Fig. 2 show that the different types of interfering echoes are characterized by different shapes and intensities, which will affect the type and severity of the distortions introduced in the DEER trace. The properties of these additional echoes were therefore investigated. The different types of possible echoes listed in Table 1 were investigated separately by recording transients for two- and three-pulse sequences containing only the pulses that generate them. For example, the primary echo $\mathrm{PE}_{1 \mathrm{p}}$ was recorded using a two pulse sequence with the first pulse corresponding to the $\frac{\pi}{2}$ pulse of the DEER sequence and the second pulse corresponding to the $\pi$ pump pulse of the DEER sequence. The 'pump pulse' in these sub-sequences was either a monochromatic rectangular pulse or a sech/tanh pulse with the same properties as the pump pulse used in the DEER experiment. The corresponding echoes were simulated using a density matrix approach taking the instrumental transfer function into account for both excitation and detection (see Sections 5 and 5.3 and ESI $\uparrow$ for details). The experimental and simulated echo transients are compared in Fig. 4. Fourier transforms of the different echoes are shown in the ESI $\dagger$ in Fig. S7. 


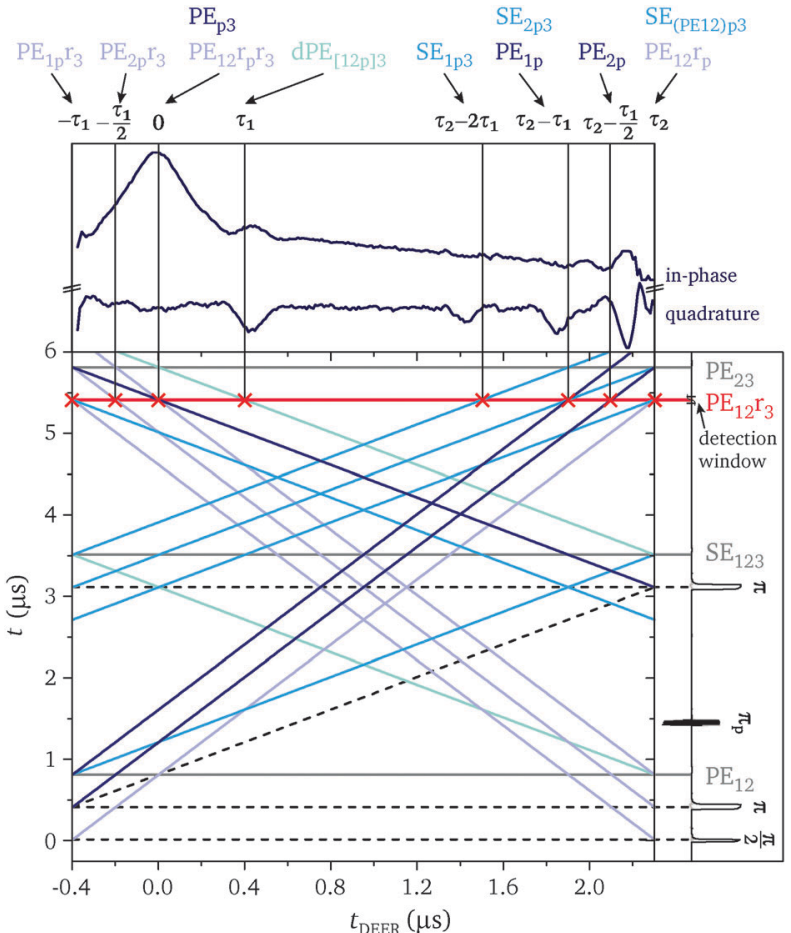

Fig. 3 Positions of the echo crossings in a four-pulse DEER experiment with $\tau_{1}=400 \mathrm{~ns}$ and $\tau_{2}=2.3 \mu \mathrm{s}$. The four-pulse DEER sequence is plotted along the $y$-axis with pulse positions represented by dashed lines. The standing echoes are shown in grey and the different types of moving echoes induced by the pump pulse are shown in different shades of blue (see Table 1 for details on the corresponding coherence transfer pathways and echo times). The crossing positions of the moving echoes with the detected refocused echo $\left(\mathrm{PE}_{12} \mathrm{r}_{3}\right.$, position shown in red) are highlighted. In the absence of phase cycling, the echo crossings lead to artefacts in the DEER trace as shown for the experimental data of Fig. 2 in the top panel.

2.2.1 Monochromatic rectangular pulses. The echoes generated by the observer sequence are shown in Fig. $4 \mathrm{~A}$. The shapes of the echoes are well reproduced by the simulations. The difference in relative intensities of the stimulated and refocused echoes in the three-pulse sequence is due to the neglect of relaxation in the simulations. An accurate reproduction of the experimental echo shapes required the inclusion of the effect of the resonator profile for both excitation and detection. In particular, taking the resonator into account leads to the observed asymmetry in the $\mathrm{PE}_{23}$ echo generated by two consecutive $\pi$ pulses. The negative and positive parts of this echo are due to the consecutive refocusing of the counter-rotating magnetizations of spin packets with positive or negative frequency offsets with respect to the observer frequency along the negative $x$-axis and the positive $x$-axis. The two $\pi$ pulses do not generate transverse magnetization for spin packets on resonance. The shape of the stimulated echo $\mathrm{SE}_{123}$ is due to a similar effect.

In two-pulse sequences with monochromatic rectangular pulses at two different frequencies (see Fig. 4B), echoes are generated by spin packets resonantly excited by both pulses. Their intensity envelope and frequency content is therefore determined by the extent of overlap of the pulse excitation profiles (see ESI $\dagger$ ). The excitation profile of an ideal rectangular inversion pulse can be approximated by a sinc function

$$
\frac{M_{z}}{M_{0}}=1-(1-\cos \theta)\left[\frac{\sin \left(\pi \Delta \nu t_{\mathrm{p}}\right)}{\left(\pi \Delta \nu t_{\mathrm{p}}\right)}\right]^{2}
$$

where $\theta$ is the flip angle and $t_{\mathrm{p}}$ the length of the pulse. The corresponding transverse component of the magnetization can be calculated as $\sqrt{M_{x}^{2}+M_{y}^{2}}=\sqrt{1-M_{z}^{2}}$. Instrumental distortions lead to attenuation of the side lobes (wings) of the sinc function. ${ }^{28}$ Nevertheless, the overlap of the excitation profiles of a $32 \mathrm{~ns}$ or a $64 \mathrm{~ns}$ pulse at the observer frequency and a $64 \mathrm{~ns}$ pulse at a pump frequency with a frequency offset of $70 \mathrm{MHz}$ is sufficient to generate primary echoes $\mathrm{PE}_{1 \mathrm{p}}$ and $\mathrm{PE}_{2 \mathrm{p}}$ of considerable intensity (see for example Fig. 2, panel 4). The main frequency component of the primary echoes $\mathrm{PE}_{1 \mathrm{p}}$ and $\mathrm{PE}_{2 \mathrm{p}}$ is centred at $70 \mathrm{MHz}$, i.e. due to spin packets at the frequency of the pump pulse, with some contributions from spin packets with lower resonance frequencies (see ESI, $\dagger$ Fig. S7). The main deviations of the simulated echo shape from the experimental one are due to a lower relative weight of higher-frequency components in the experimental echoes (see discussion below). The primary echo $\mathrm{PE}_{\mathrm{p} 3}$ generated by a $\pi$ pulse at the pump frequency and followed by a $\pi$ pulse at the observer frequency is mainly due to spin packets resonant with the observer frequency, whose magnetization has been tilted away from the $z$-axis by the pulse at the pump frequency.

The stimulated and refocused echoes generated in threepulse sequences where one of the three pulses is applied at a different frequency are also shown in Fig. 4B. The stimulated and refocused echoes are shown on the same scale and reveal a significant reduction in intensity of the refocused echo compared to the corresponding stimulated echo. The refocused echoes are generally within the noise and, under the present experimental conditions, will not introduce artefacts in the DEER trace when they cross the detection window. The relative intensity of the two types of three-pulse echoes is relatively well reproduced by the simulations. The slightly higher intensities of the refocused echoes in the simulations are due to the neglect of spin relaxation. The positions of the different features in the simulated echo shapes also agree remarkably well with experiment, however differences in their relative intensities are observed. Again, the discrepancies were found to be mainly due to different relative weights of low and high frequency components. Similarly to the $\mathrm{PE}_{\mathrm{p} 3}$ echo, the stimulated echoes for rectangular pulses at different frequencies are mainly due to spin packets with resonance frequencies close to the observer frequency.

2.2.2 Sech/tanh pulses. The echoes generated by coherence transfer pathways involving a coherent sech/tanh pump pulse $\left(t_{\mathrm{p}}=200 \mathrm{~ns}, \mathrm{BW}_{\infty}=70 \mathrm{MHz}, \beta=4 / t_{\mathrm{p}}\right.$ and $\left.\Delta \nu=75 \mathrm{MHz}\right)$ are shown in Fig. 4C. An ideal adiabatic sech/tanh pulse with a high $\beta$ parameter approaches a rectangular inversion profile with sharp edges and therefore provides uniform inversion within the chosen bandwidth. In EPR, in order to achieve inversion over a given bandwidth for a relatively short pulse (100 to $200 \mathrm{~ns}$ ) 
A Observer sequence echoes
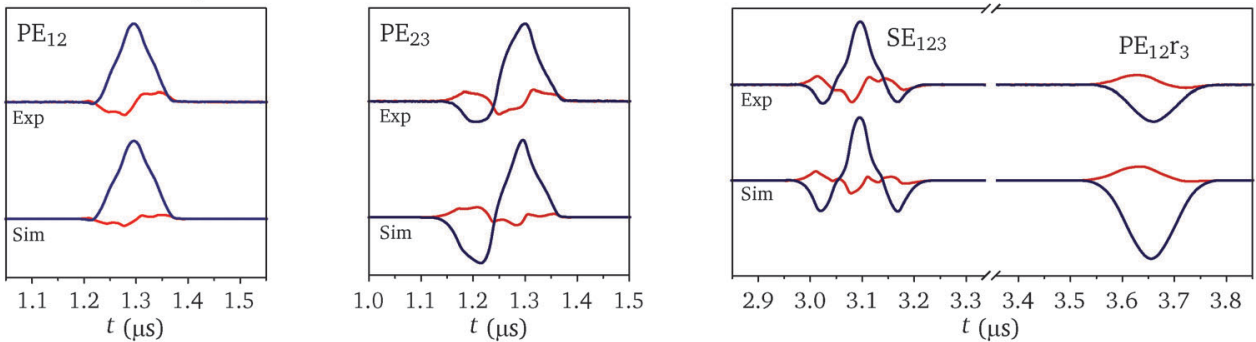

$\begin{array}{lllllllllll}2.9 & 3.0 & 3.1 & 3.2 & 3.3 & 3.4 & 3.5 & 3.6 & 3.7 & 3.8\end{array}$ $t(\mu s)$

B Additional echoes for a coherent rectangular pulse at the pump frequency
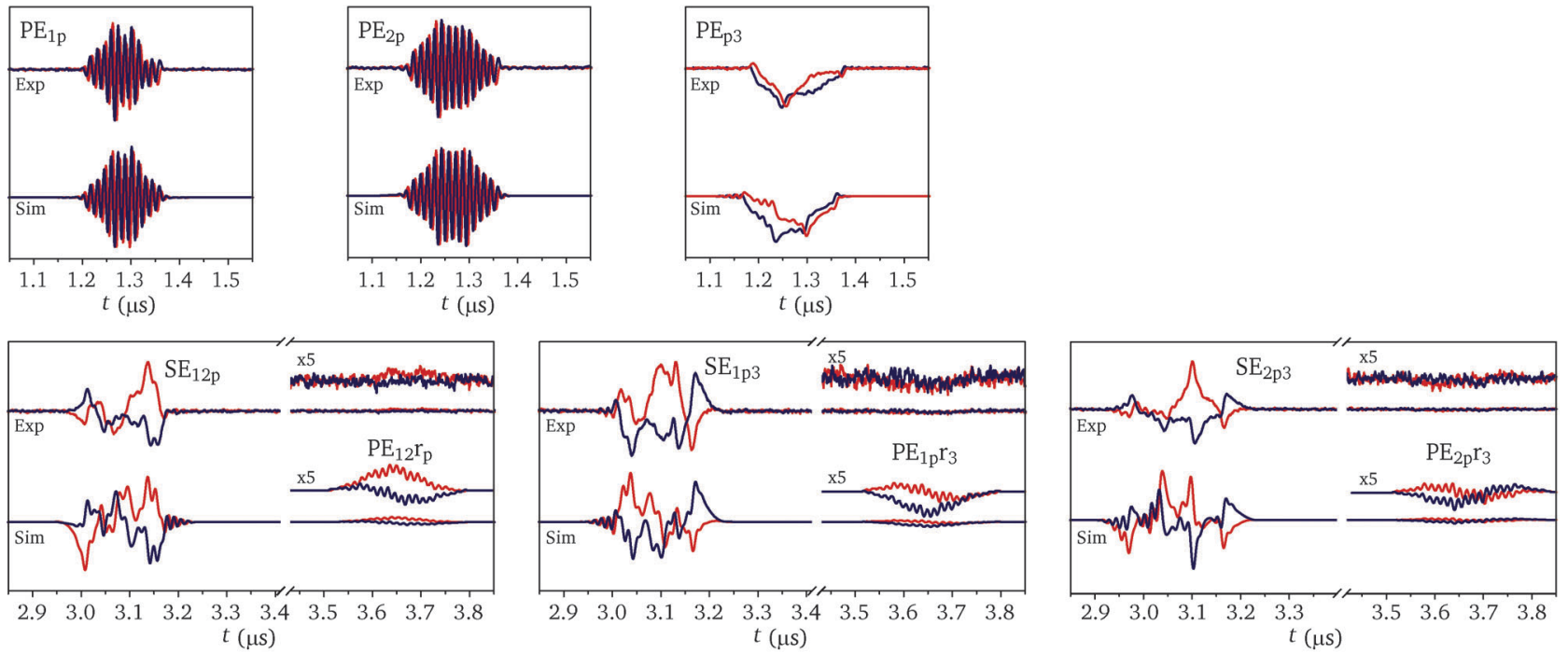

C Additional echoes for a coherent sech/tanh pulse at the pump frequency
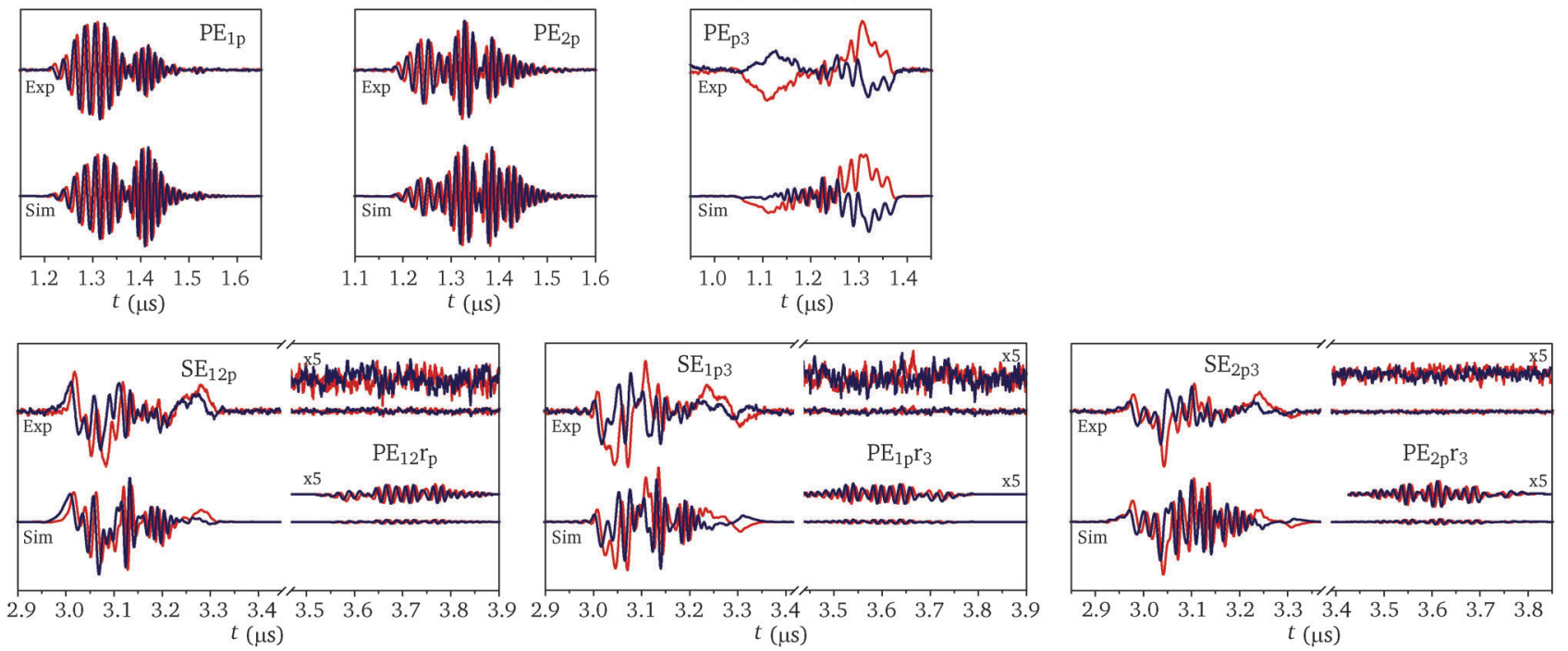

Fig. 4 Experimental (top) and simulated (bottom) two- and three-pulse echoes generated by $32 \mathrm{~ns}$ and $64 \mathrm{~ns}$ rectangular $\frac{\pi}{2}$ and $\pi$ pulses at the observer frequency (A), by rectangular pulses at the observer frequency and a $64 \mathrm{~ns}$ rectangular $\pi$ pulse at a coherent pump frequency $(\Delta \nu=+70 \mathrm{MHz})(\mathrm{B})$, and by rectangular pulses at the observer frequency and a $\left.200 \mathrm{~ns} \mathrm{sech/tanh} \mathrm{pulse} \mathrm{(BW})_{\infty}=70 \mathrm{MHz}, \beta=4 / t_{\mathrm{p}}\right)$ at a coherent pump frequency $(\Delta \nu=+75 \mathrm{MHz})(\mathrm{C})$. The time axis $t$ indicates the time from the start of the pulse sequence. The in-phase and quadrature components are shown in blue and red, respectively. The echoes are labelled as in Table 1, where 1, 2 and 3 stand for the first $\left(\frac{\pi}{2}\right)$, second $(\pi)$ and third $(\pi)$ observer pulse of a four-pulse DEER sequence. Twopulse echoes were recorded and simulated with a delay $\tau$ equal to $600 \mathrm{~ns}$, three-pulse echoes with a first delay of $600 \mathrm{~ns}$ and a second delay of $1800 \mathrm{~ns}$. The simulations were performed as described in the main text and the time axis, intensity and phase were adjusted individually to match the experiment.

and limited power, the sech/tanh pulse is typically truncated $\left(\beta \approx 4 / t_{\mathrm{p}}\right)$ without significantly affecting performance. ${ }^{16,29}$
This does however lead to reduced inversion uniformity and decreased selectivity (decreased steepness of the edges of the 
inversion profile), and therefore to an increased overlap of the excitation profiles of the pump and observer pulses.

Similarly to the case of the rectangular pump pulse, the frequency content of the primary echoes $\mathrm{PE}_{1 \mathrm{p}}$ and $\mathrm{PE}_{2 \mathrm{p}}$ for a sech/tanh pump pulse is determined by the overlap of the pulse excitation profiles. Fourier transformation of the echoes shows that the main frequency components occur at the positions of the sinc wings of the rectangular observer pulses within the excitation bandwidth of the sech/tanh pulse (see ESI, $\dagger$ Fig. S7). Since the sech/tanh pulse flips spins with different resonance frequencies at different times, the refocusing also occurs at different times, leading to the appearance of 'chirped' echoes. The absence of frequency components corresponding to the zero crossings causes the apparent separation of the echo into three or four components (for $\mathrm{PE}_{1 \mathrm{p}}$ and $\mathrm{PE}_{2 \mathrm{p}}$, respectively). The primary echo $\mathrm{PE}_{\mathrm{p} 3}$ is again mainly determined by spin packets with resonance frequencies close to the observer frequency. Spin packets with lower resonance frequencies contribute to the echo mainly at later times, since in this case the sech/tanh pulse precedes the $\pi$ pulse at the observer frequency.

In analogy to the results obtained for a rectangular pulse at the pump frequency, the stimulated echoes generated by a three-pulse sequence involving a sech/tanh pulse are characterized by a much higher intensity compared to the corresponding refocused echoes (see bottom panels of Fig. 4C). The three types of stimulated echoes contain multiple frequency components at frequencies ranging from the observer frequency to the upper limit of the sech/tanh pulse frequency sweep. The simulations are again in relatively good overall agreement with experiment, they however overestimate the intensity of higher-frequency components. This can be seen most clearly for the primary echoes $\mathrm{PE}_{1 \mathrm{p}}$ and $\mathrm{PE}_{2 \mathrm{p}}$, where the higher frequency components in the experimental echoes, contributing at later times of the echo, are clearly attenuated with respect to the simulations. Experiments performed with an inverted sweep direction of the sech/tanh pulse demonstrate that the observed attenuation of the echo signal at longer times is not due to relaxation. Since the excitation bandwidth of the resonator and the detection bandwidth of the video amplifier have been taken into account in the simulations, the remaining discrepancies are most likely due to an oversimplification in the modelling of the transfer functions or to additional distortions of the pulses in the transmitter (see ESI, $\dagger$ Section 2.3 for further discussion). Contributions from relaxation effects, e.g. spectral diffusion and instantaneous diffusion, can also not be excluded.

2.2.3 Gaussian observer pulses. Since the intensity of echoes generated by combinations of observer and pump pulses depends on the extent of overlap of their excitation profiles, the use of Gaussian observer pulses could potentially lead to a reduction of the artefacts observed in DEER with a coherent sech/tanh pump pulse. A Gaussian pulse is more selective than a rectangular pulse, as the excitation profile is also a Gaussian and thus the sinc wings are absent. Two- and three-pulse echoes measured for analogous pulse sequences as shown in Fig. 4C but with the rectangular observer pulses replaced by Gaussian pulses with the same excitation bandwidth are shown in Fig. 5. As expected, the intensity of the primary echoes for sequences with a sech/tanh refocusing pulse at $\nu_{\text {pump }}$ is reduced almost to the noise level. However, the primary echo $\mathrm{PE}_{\mathrm{p} 3}$ and the stimulated echoes are still characterized by an appreciable intensity. While a series of spin packets at different frequencies within the excitation bandwidths of both observer and pump pulses contributed to these echoes in the case of rectangular observer pulses, here they are determined almost exclusively by spin packets with frequencies close to the observer frequency. This indicates that, even though the excitation profiles of the pulses do not overlap, the off-resonant effect of the sech/tanh pump pulse on spin packets at the observer frequency is sufficient to generate measurable echoes. Therefore, the use of Gaussian observer pulses does not significantly improve the suppression of echocrossing artefacts.

2.2.4 Vector model for off-resonant echo formation. The formation of echoes by pulses at different frequencies is related to the resonance line shifts and phase shifts induced by off-resonant microwave radiation. ${ }^{10,18,30-32}$ The dynamic phase shifts in the observer echo caused by the pump pulse in DEER experiments have been analysed in detail and explained using a simple vector model by Bowman and Maryasov. ${ }^{10}$ The same

Echoes for Gaussian observer pulses and a coherent sech/tanh pulse at the pump frequency
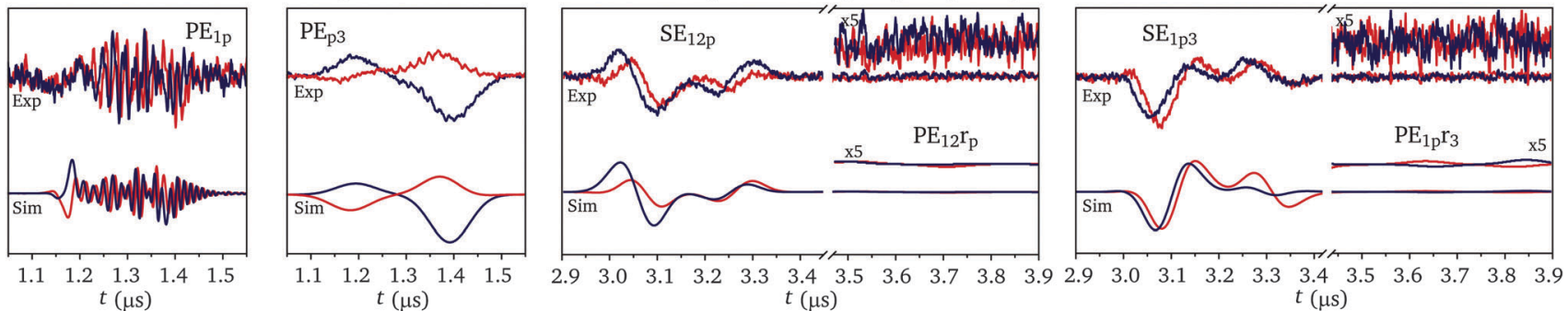

Fig. 5 Experimental (top) and simulated (bottom) two- and three-pulse echoes generated by Gaussian observer pulses, with pulse lengths of 80 ns and 160 ns and FWHM of 32 ns and 64 ns for $\frac{\pi}{2}$ and $\pi$ pulses, respectively, and a $200 \mathrm{~ns}$ sech/tanh pulse $\left(\mathrm{BW}_{\infty}=70 \mathrm{MHz}, \beta=4 / \mathrm{t}_{\mathrm{p}}\right)$ at a coherent pump frequency $(\Delta \nu=+75 \mathrm{MHz}$ ). The echoes are labelled as in Table 1. Two-pulse echoes were recorded and simulated with a delay $\tau$ equal to $600 \mathrm{ns,}$ three-pulse echoes with a first delay of $600 \mathrm{~ns}$ and a second delay of $1800 \mathrm{~ns}$. The simulations were performed as described in the main text. The time axis, intensity and phase were adjusted individually to match the experiment. The complete set of echoes for Gaussian observer pulses is shown in Fig. S8 in the ESI. $\dagger$ 
vector model can also be used to explain the formation of the echoes described above: a pulse applied to a spin system at thermal equilibrium tilts the magnetization of off-resonant spin packets with respect to the $z$-axis. After the pulse, there is therefore a transverse component of the magnetization, which defocuses and can be refocused to an echo by a pulse at the resonance frequency of the corresponding spin packet, leading to a primary echo with the main frequency components corresponding to the frequency of the refocusing pulse. The formation of the stimulated echoes can be similarly explained.

Simulations performed for different observer pulse lengths and frequency offsets have shown that the integrated intensity of refocused echoes generated by two rectangular pulses at the observer frequency and a third sech/tanh pulse centred at the pump frequency is approximately proportional to the overlap of the corresponding excitation profiles (see ESI, $\dagger$ Section 2.5). The integrated intensity of the corresponding stimulated echo, on the other hand, decays more slowly for decreasing excitation overlap. The experiments described in this paper were performed with a sufficiently small overlap of the excitation profiles of observer and pump pulses to render the intensity of the echoes refocused by both a pulse at $\nu_{\text {obs }}$ and a pulse at $\nu_{\text {pump }}$ negligible. In case of increased overlap of the excitation profiles due to shorter observer pulses, increased pump pulse bandwidth or smaller frequency offset, these refocused echoes can gain intensity. Similarly, an increase in intensity is also possible in the presence of a significant contribution of microwave radiation at the observer frequency during the pump pulse due to, for example, LO leakage at the IQ mixer. It is important to consider that the relative magnitude of the LO leakage compared to the desired signal will increase in cases where the amplifier is run in compression.

\subsection{Echo crossing artefacts in the four-pulse DEER trace}

In general, the properties of the coherent pump pulse and the offset between observer and pump frequency will determine the intensity, shape and frequency content of the additional echoes and therefore the nature of the corresponding artefacts in the DEER trace. The length of the pulses determines the length of the echoes and therefore the extent of the regions in the DEER trace affected by echo crossing artefacts. Depending on the dipolar frequency modulating the refocused echo, these artefacts could be difficult to identify as such and lead to errors in distance distributions determined by Tikhonov regularization.

The intensity of the interfering echoes depends on the excitation bandwidth overlap between observer and pump pulses. An increased overlap will lead to an increased intensity of the interfering echoes and therefore to more pronounced distortions in the DEER trace, in addition to the appearance of a '2 + 1'-type dipolar modulation of the refocused echo $\mathrm{PE}_{12} \mathrm{r}_{3}$ towards the end of the DEER trace. ${ }^{11,12}$ For the $32 \mathrm{~ns} \frac{\pi}{2}$ and $64 \mathrm{~ns} \pi$ observer pulses used here and a $64 \mathrm{~ns} \pi$ pump pulse at a frequency offset of $70 \mathrm{MHz}$, or a $200 \mathrm{~ns}$ sech/tanh pump pulse sweeping over a frequency range of 40 to $110 \mathrm{MHz}$ (centred at $75 \mathrm{MHz}$ ) with respect to the observer frequency, the effects of the crossing echoes are clearly seen in the DEER trace (see Fig. 2). At a central frequency offset of $95 \mathrm{MHz}$ for the $\mathrm{sech} /$ tanh pulse, the distortions were much reduced, but still present (see ESI, $\uparrow$ Fig. S10). Increasing the frequency offset, in this case by shifting the observer frequency to maintain the same pumping efficiency, leads to decreased intensity of the recorded echo and therefore a decreased signal-to-noise ratio. Increasing the length of the rectangular pulses and decreasing the bandwidth of the sech/tanh pulse would reduce the excitation bandwidth overlap, however, this would also lead to increased orientation selection in the first case and additionally to a reduced modulation depth in the second case. Therefore, for DEER measurements between nitroxides at X- or Q-band, a reduction of the excitation bandwidth overlap of observer and pump pulse sufficient to remove the effect of the interfering echoes is not feasible.

Due to the contributions at different frequencies, another possibility of reducing the echo crossing artefacts in the DEER trace could be the use of a detection filter. While a low-pass filter (e.g. $20 \mathrm{MHz}$ ) will reduce the artefacts due to the primary echoes $\mathrm{PE}_{1 \mathrm{p}}$ and $\mathrm{PE}_{2 \mathrm{p}}$ dominated by high-frequency components, it will however not have a significant impact on the artefacts due to the remaining primary and the stimulated echoes with their significant contribution at baseband.

\subsection{Phase cycling in four-pulse DEER}

The artefacts introduced in the DEER trace by interfering echoes generated by the coherent pump pulse can be removed by phase cycling. We generated different types of nested phase cycles and analysed them in terms of their effectiveness in suppressing undesired coherence transfer pathways following the theory described in ref. 26 and 27. In the following, different types of phase cycling schemes will be referred to with short-hand notation, ${ }^{27}$ where the initial phase is indicated for each pulse and round parentheses and square brackets indicate cycles in $180^{\circ}$ and $90^{\circ}$ steps, respectively (see Table 2). The subscript $\mathrm{p}$ indicates that the corresponding pulse is a pump pulse.

DEER traces recorded with different phase cycles are compared in Fig. 6 for both monochromatic and sech/tanh pump pulses. The two-step phase cycle on the first observer pulse, $(x) x x_{\mathrm{p}} x$, which is typically used for four-pulse DEER with an incoherent pump pulse channel, only removes the echo crossing artefacts at $t_{\mathrm{DEER}}=0, t_{\mathrm{DEER}}=\tau_{2}-\frac{1}{2} \tau_{1}$ and $t_{\mathrm{DEER}}=\tau_{2}-\tau_{1}$ due to primary echoes $\mathrm{PE}_{\mathrm{p} 3}$ and $\mathrm{PE}_{2 \mathrm{p}}$ and the stimulated echo $\mathrm{SE}_{2 \mathrm{p} 3}$, respectively. A four-step phase cycle $(x)(x) x_{\mathrm{p}} x$, where the phase of the second observer pulse is also varied, also removes the delayed primary echo $\mathrm{PE}_{[12 \mathrm{p}] 3}$ crossing at $t_{\mathrm{DEER}}=\tau_{1}$. The eight-step phase cycle $(x)[x] x_{\mathrm{p}} x$ (see Table 2 for details) also removes the stimulated echo $\mathrm{SE}_{1 \mathrm{p} 3}$ at $t_{\mathrm{DEER}}=\tau_{2}-2 \tau_{1}$ and the primary echo $\mathrm{PE}_{1 \mathrm{p}}$ at $t_{\mathrm{DEER}}=\tau_{2}-\tau_{1}$. This phase cycle therefore removes all visible artefacts in the four-pulse DEER traces shown in Fig. 6. However, it does not suppress the refocused echo $\mathrm{PE}_{12} \mathrm{r}_{\mathrm{p}}$ and the stimulated echo $\mathrm{SE}_{(\mathrm{PE} 12) \mathrm{p} 3}$ at $t_{\mathrm{DEER}}=\tau_{2}$, and the twice refocused echo $\mathrm{PE}_{12} \mathrm{r}_{\mathrm{p}} \mathrm{r}_{3}$ at $t_{\mathrm{DEER}}=0$. The echo crossings at $t_{\text {DEER }}=\tau_{2}$ should in general not be a problem, since they fall within the region of pulse overlap that is typically discarded. 
Table 2 Phase cycles for the four-pulse, the two versions of the five-pulse and the seven-pulse DEER experiments

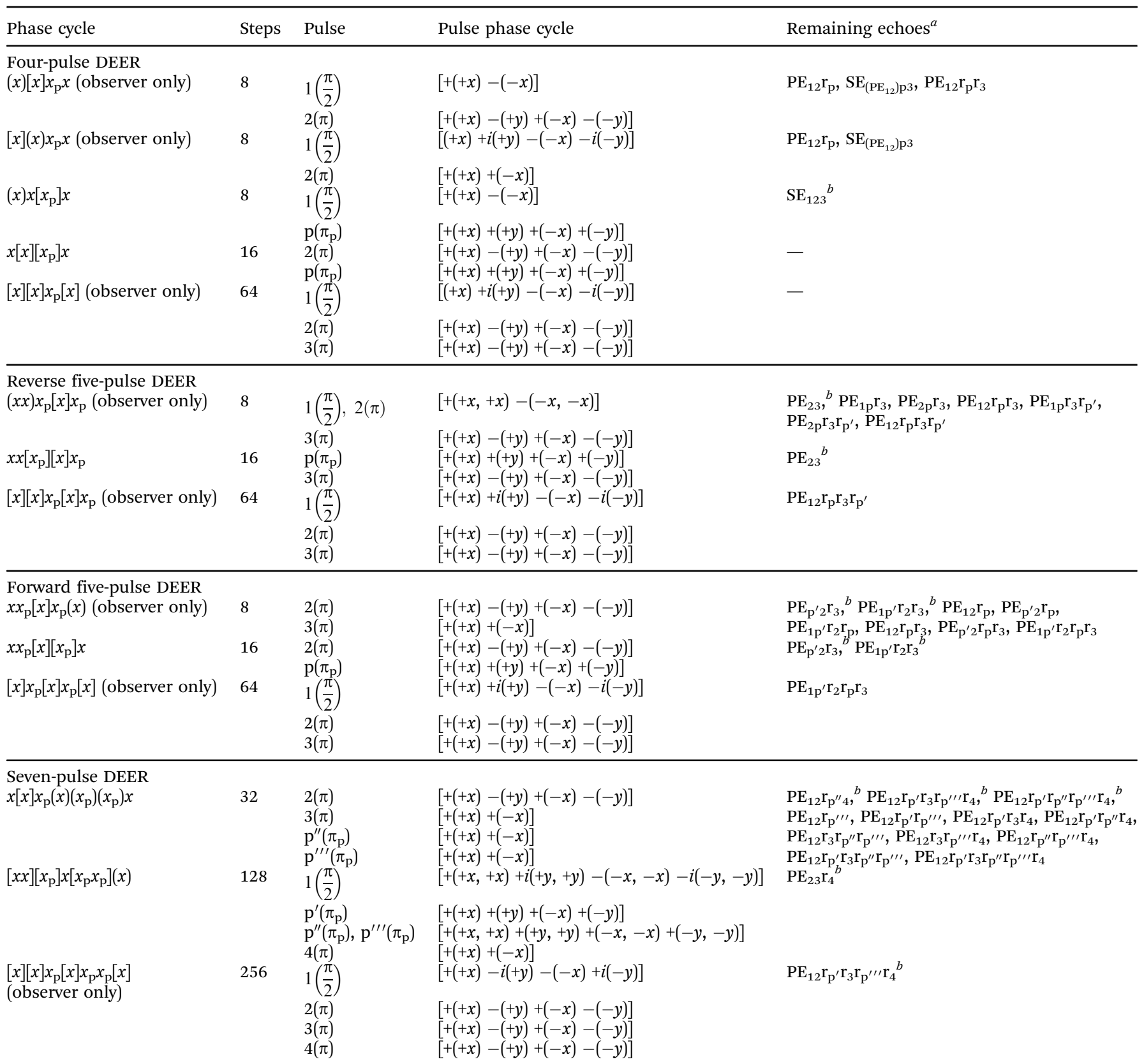

${ }^{a}$ Standing echoes within about $\tau_{1}$ from the detected refocused echo and echoes crossing the refocused echo in the range $0 \leq t_{\mathrm{DEER}} \leq t_{\mathrm{DEER}, \mathrm{max}}$, where $t_{\mathrm{DEER}, \max }$ is the maximum evolution time up to overlap of observer and pump pulses. (See ESI for details). ${ }^{b}$ Standing echo.

However, the echo crossing at $t_{\mathrm{DEER}}=0$ could interfere with the exact determination of the zero-time or in some cases even with the analysis of short distances, if the experimental conditions lead to non-negligible intensity of the refocused echo $\mathrm{PE}_{12} \mathrm{r}_{\mathrm{p}} \mathrm{r}_{3}$. As discussed in Section 2.2.4, the refocused echoes become significant in case of substantial observer and pump pulse excitation profile overlap or in the presence of a significant contribution at the observer frequency during the pump pulse. The eight-step phase cycle $[x](x) x_{\mathrm{p}} x$ (Table 2) also removes the potential contribution at $t_{\mathrm{DEER}}=0$, leaving only echoes crossing at $t_{\mathrm{DEER}}=-\tau_{1}$ and $t_{\mathrm{DEER}}=\tau_{2}$, and is therefore a better phase cycle for the four-pulse experiment.
The phase cycles discussed so far only cycle the phases of the observer pulses, however in a fully coherent spectrometer, phase cycling of the pump pulse can also be used. The same relationships between changes in pulse phase and resulting phase shifts of the signal apply to both observer and pump pulses. Potential phase cycles can therefore be devised following the same procedure. ${ }^{27}$ Addition of a four-step phase cycle on the pump pulse to the standard two-step phase cycle on the first observer pulse, $(x) x\left[x_{\mathrm{p}}\right] x$, removes all crossing echoes, but leaves the standing stimulated echo $\mathrm{SE}_{123}$, which could interfere with detection if $\tau_{1} \approx \tau_{2}$. The 16-step phase cycle $x[x]\left[x_{\mathrm{p}}\right] x$ removes all echoes except for the primary echo $\mathrm{PE}_{12}$ 

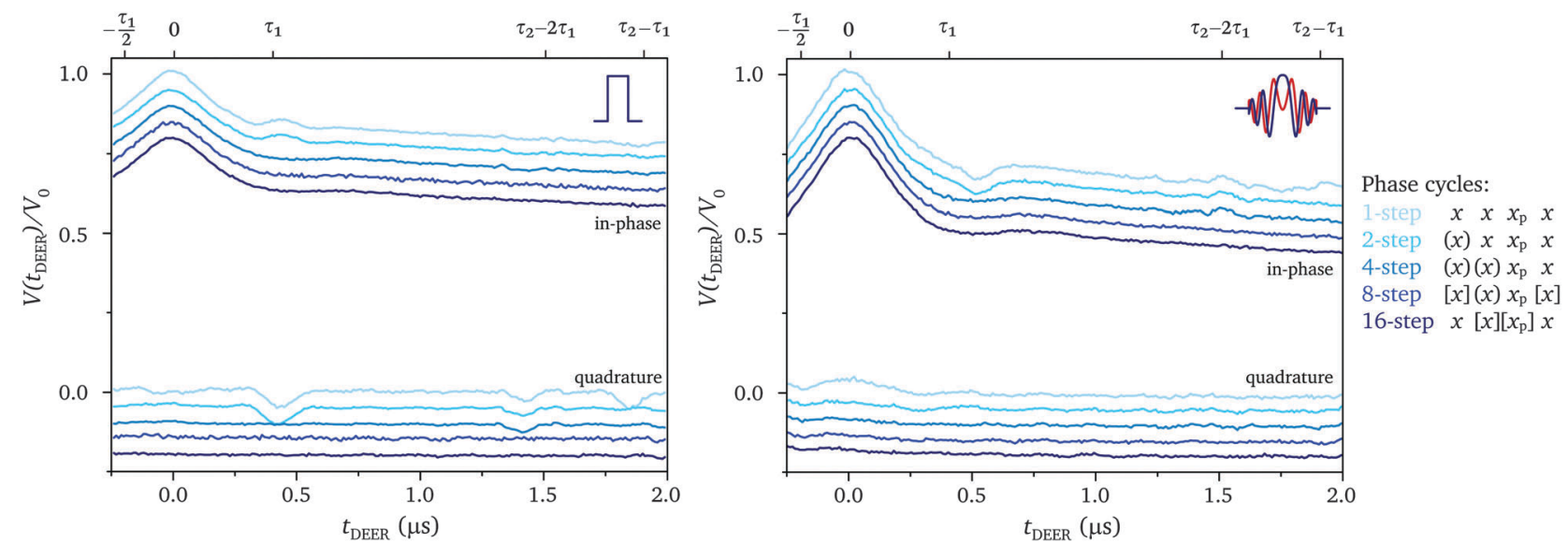

Fig. 6 Four-pulse DEER traces recorded with different phase cycles for a monochromatic rectangular pump pulse (left, $t_{\mathrm{p}}=64 \mathrm{~ns}, \Delta \nu=70 \mathrm{MHz}$ ) and a sech/tanh pump pulse (right, $t_{\mathrm{p}}=200 \mathrm{~ns}, \mathrm{BW} \mathrm{\infty}_{\infty}=70 \mathrm{MHz}, \beta=4 / t_{\mathrm{p}}, \Delta \nu=75 \mathrm{MHz}$ ). Traces recorded with different phase cycles are slightly offset for better comparison. In both cases, the observer pulse lengths were $t_{\pi / 2}=32 \mathrm{~ns}$ and $t_{\pi}=64 \mathrm{~ns}$ and inter-pulse delays were set to $\tau_{1}=400 \mathrm{~ns}$ and $\tau_{2}=2.3 \mu \mathrm{s}$. Further experimental details can be found in Section 5. Remaining distortions at the end of the trace were found to be due to instrumental effects due to the close proximity of the pump and the last observer pulse.

and the desired refocused echo $\mathrm{PE}_{12} \mathrm{r}_{3}$ (Fig. 7). Achievement of a similarly efficient suppression of coherence transfer pathways using only phase cycling of the observer pulses would require a 64-step phase cycle, where each of the observer pulses is cycled in steps of $90^{\circ}\left(\right.$ CYCLOPS $\left.^{33}\right)$, i.e. $[x][x] x_{\mathrm{p}}[x]$.

In the presence of a contribution at the observer frequency during the pump pulse, as resulting, for example, due to LO leakage at the IQ mixer or incorrect calibration of the DC offsets of the two AWG channels in an IQ upconversion setup (see Section 5), this resonant contribution can also lead to the formation of the same types of echoes. The echoes are in this case due to spin packets resonant with $\nu_{\mathrm{obs}}$, and therefore the refocused echoes, otherwise of negligible intensity, could become significant. Since the phase of the contribution at $\nu_{\mathrm{obs}}$
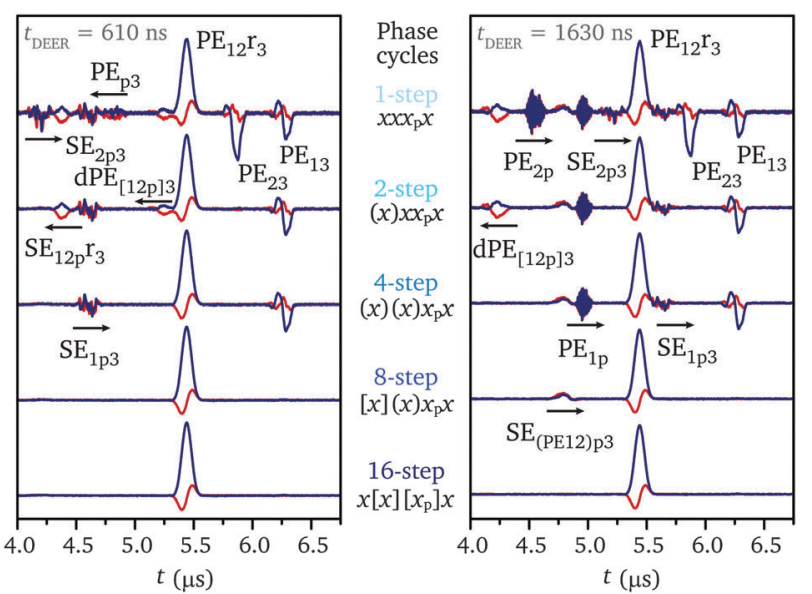

Fig. 7 Echo transients recorded for two different positions of a monochromatic rectangular pump pulse ( $t_{\text {DEER }}=610 \mathrm{~ns}$ (left) and $t_{\text {DEER }}=1630 \mathrm{~ns}$ (right)) for different phase cycles of the four-pulse DEER experiment. The experimental parameters are the same as reported in Fig. 6 . The different echoes are labelled according to the notation of Table 1 and the arrows indicate their direction of movement. is not affected by the phase of the AWG programmed waveform, phase cycling of the pump pulse only leads to the elimination of the echoes created by the pump pulse, but not of the echoes due to the constant contribution at $\nu_{\mathrm{obs}}$. Phase cycling of the observer pulses removes both types of echoes, therefore phase cycles only involving the observer pulses should be used if leakage of microwaves at the observer frequency is a significant concern. $\$$

In conclusion, the suppression of echo crossing artefacts within the time window of interest in the four-pulse DEER experiment requires the use of an eight-step phase cycle, either only involving the observer pulses, $[x](x) x_{\mathrm{p}} x$, or including pump pulse phase cycling, $(x) x\left[x_{\mathrm{p}}\right] x$.

\section{Multi-pump-pulse DEER}

DEER experiments involving multiple pump pulses and additional refocusing observer pulses have been proposed with the aim to extend the accessible dipolar evolution window and allow measurements of longer inter-spin distances. ${ }^{14}$ Five-pulse and seven-pulse DEER experiments, with two and three pump pulses, respectively, have been demonstrated to lead to improved sensitivity in the measurement of long distances. ${ }^{14,16,21}$ The increasing number of pulses in these experiments increases the number of coherence transfer pathways that could potentially lead to the formation of echoes crossing the detection window. The echoes introduced by coherent observer and pump pulses in these extended DEER experiments are analysed in this section and phase cycling schemes for their removal are examined.

In addition to the echo crossing artefacts, the multi-pumppulse DEER experiments are generally also affected by artefacts arising from residual signals due to undesired population transfer pathways involving B spins excited by only a subset

$\ddagger$ If all pulses are programmed on the AWG and affected by the same amount of LO leakage, echoes induced by the leaked observer frequency only could still be formed, but their intensities should be relatively low. 
of the pump pulses. ${ }^{14,21}$ In the case of the five-pulse DEER experiment, in addition to the desired pathway leading to the five-pulse signal, the pathway corresponding to B spins not excited by the second pump pulse $\left(\mathrm{p}^{\prime}\right)$ leads to a residual four-pulse signal with a zero-time $t_{\mathrm{DEER}}=\tau_{1}-t_{2}$ (or $t_{\mathrm{DEER}}=$ $\tau_{2}-t_{2}$ in reverse five-pulse DEER). Similarly, in the sevenpulse DEER experiment performed as described here, two five-pulse pathways involving only a single pump pulse can lead to residual contributions with zero-points at $t_{1}=\tau_{1}$ and $t_{3}=\tau_{3}$, corresponding to $t_{\mathrm{DEER}}=-\tau_{1}+\tau_{2}+\tau_{3}-t_{2}$ and $t_{\mathrm{DEER}}=$ $\tau_{1}+\tau_{2}-\tau_{3}-t_{2}{ }^{21}$

The artefacts introduced by these additional population transfer pathways cannot be removed by phase cycling. The intensity of the corresponding signals can be reduced by optimizing the pump pulses to give population transfer amplitudes close to unity. Different strategies for the extraction of the signal of interest have been proposed, if the artefacts cannot be removed experimentally. ${ }^{14,21}$ These strategies are based on the subtraction of a reference signal, either recorded experimentally or reconstructed based on the experimental multipump-pulse DEER trace. If the original experimental DEER data contains echo crossing artefacts, these artefacts are propagated during the correction procedures resulting in a final signal with even more significant distortions over more extensive regions of the trace.

\subsection{Five-pulse DEER}

In five-pulse DEER, a second pump pulse is used to shift the position of dipolar refocusing from $t_{1}=\tau_{1}$ in the fourpulse DEER experiment to $t_{1}=t_{2}$, giving the time axis for the experiment $t_{\mathrm{DEER}}=t_{1}-t_{2}$, where $t_{2}$ is the delay between the second pump pulse and the second or third observer pulse. The dipolar evolution time is thus extended up to $\tau_{1}+\tau_{2}-t_{2}{ }^{14}$ The two pump pulses have opposite frequency sweep directions to remove the offset-dependence of dipolar refocusing. The inter-pulse delays $\tau_{1}$ and $\tau_{2}$ are chosen to be equal or almost equal, leading to an increase in the effective relaxation time $T_{\mathrm{m}}$ by minimizing nuclear spin diffusion. ${ }^{14}$ Two different versions of this experiment have been proposed, with the second pump pulse either preceding the second observer pulse or following the last observer pulse (see Fig. 1) ${ }^{14,16}$ Here, the two versions of the experiment will be referred to as forward and reverse fivepulse DEER, respectively.

The five pulses lead to $3^{N-1}=81$ coherence transfer pathways, starting from thermal equilibrium and ending with observer spin coherence order -1 . Among these, 19 generate standing echoes and 46 moving echoes (see ESI, $\uparrow$ Tables S1 and S2 for a list of echoes, the corresponding coherence transfer pathways and refocusing points). The positions of the echoes during a reverse five-pulse DEER experiment with the interpulse delays $\tau_{1}=2.1 \mu \mathrm{s}$ and $\tau_{2}=2.4 \mu \mathrm{s}$ are shown in Fig. 8A. There are 32 echoes crossing the detection window in the relevant range $0 \leq t_{\mathrm{DEER}} \leq \tau_{1}+\tau_{2}-t_{2}$. The crossing positions are clustered around $\frac{1}{2} \tau_{1}, \tau_{1}, \frac{1}{2} \tau_{1}+\tau_{2}, \tau_{1}+\tau_{2}$ and $2 \tau_{1}$ or $2 \tau_{2}$ (for forward and reverse five-pulse DEER, respectively) and separated by multiples of $\frac{1}{2} t_{2}$. In addition to this, there are

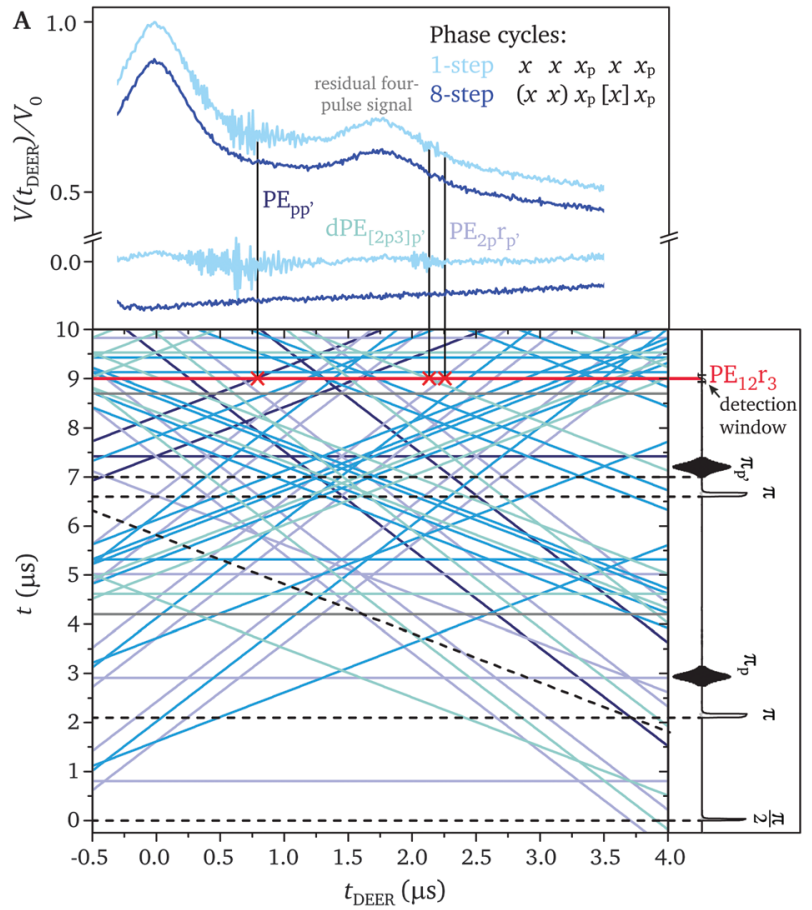

B

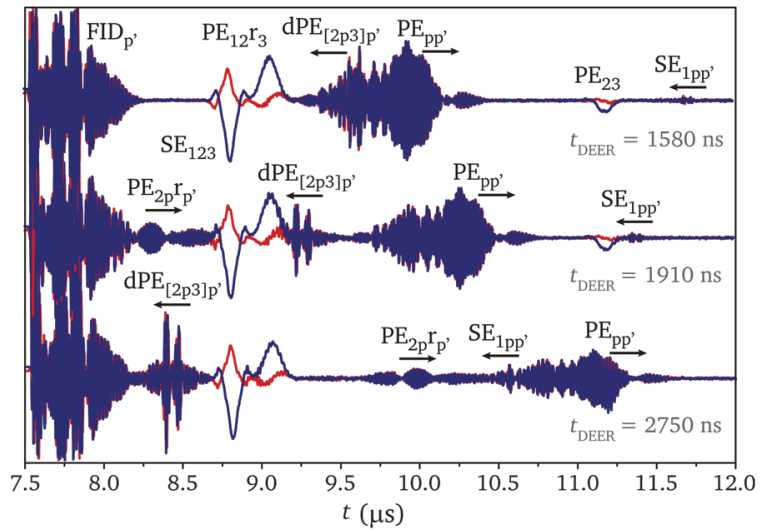

Fig. 8 (A) Positions of the echo crossings in a reverse five-pulse DEER experiment with the following inter-pulse delays $\tau_{1}=2.1 \mu \mathrm{s}, \tau_{2}=2.4 \mu \mathrm{s}$ and $t_{2}=400 \mathrm{~ns}$. The pulse sequence is plotted along the $y$-axis with pulse positions represented by dashed lines. The standing echoes of the observer sequence are shown in grey and the different types of echoes induced by the pump pulses are shown in different shades of blue following the same colour code as in Fig. 3 (see Table S2, ESI $\dagger$ for details on the coherence transfer pathways and echo times). Five-pulse DEER traces recorded with different phase cycles are shown in the top panel. The traces are offset to allow better comparison. The crossing positions of the most intense moving echoes with the detected refocused echo $\left(\mathrm{PE}_{12} \mathrm{r}_{3}\right.$, position shown in red) are highlighted and tentatively assigned. (B) Echo transients recorded for three positions of the moving pump pulse. The different echoes are labelled according to Table S2 (ESI $\dagger$ ) and the direction of movement for crossing echoes is indicated by arrows.

several standing echoes that can partially overlap with the detection window, depending on the difference between $\tau_{1}$ and $\tau_{2}$ and on the length of the delay $t_{2}$. In particular, if $\tau_{1}=\tau_{2}$, the stimulated echo of the observer sequence overlaps with the refocused echo and has to be removed by phase cycling. 
3.1.1 Reverse five-pulse DEER. The reverse five-pulse DEER trace recorded without phase cycling is shown in the top panel of Fig. 8A. The residual four-pulse signal at about $2 \mu$ s can be clearly identified. Significant distortions over large portions of the DEER trace can be observed in the absence of phase cycling. Inspection of echo transients recorded for different positions of the first pump pulse during the delay $\tau_{1}+\tau_{2}$ (see Fig. 8B) allows identification of the most intense echoes interfering with detection on the refocused echo $\mathrm{PE}_{12} \mathrm{r}_{3}$. The most significant artefact is caused by the primary echo generated by the two pump pulses, $\mathrm{PE}_{\mathrm{Pp}^{\prime}}$, and ranges from about $t_{\mathrm{DEER}}=200 \mathrm{~ns}$ to $t_{\mathrm{DEER}}=$ $1.2 \mu \mathrm{s}$ (predicted crossing time $t_{\text {DEER,cross }}=\tau_{2}-3 t_{2}-t_{\mathrm{p}}=0.8 \mu \mathrm{s}$ ). This echo is characterized by a similar signal intensity as the detected refocused echo and an overall echo length of about $3 t_{\mathrm{p}}=1.2 \mu \mathrm{s}$ due to the opposite sweep direction of the two pump pulses. Additional significant distortions are introduced around $t_{\mathrm{DEER}}=2.2 \mu$ s when the delayed primary echo $\mathrm{dPE}_{[2 \mathrm{p} 3] \mathrm{p}^{\prime}}$ and the refocused primary echo $\mathrm{PE}_{2 \mathrm{p}} \mathrm{r}_{\mathrm{p}^{\prime}}$ cross the detection window (predicted crossing times are $t_{\mathrm{DEER}}=\tau_{1}+t_{2}-t_{\mathrm{p}}$ and $t_{\text {DEER }}=\frac{1}{2} \tau_{1}+\tau_{2}-2 t_{2}-t_{\mathrm{p}}$, respectively). While refocused echoes involving the pump pulse as one of the refocusing pulses were found to exhibit negligible intensity in the absence of significant excitation bandwidth overlap, refocused echoes where the refocusing is induced by two pump pulses are sufficiently strong to introduce visible distortions to the DEER trace. The echo $\mathrm{PE}_{2 \mathrm{p}} \mathrm{r}_{\mathrm{p}^{\prime}}$ is due to spin packets resonant with the pump frequencies and refocused by both pump pulses. Several additional echoes cross the detection window during the dipolar evolution time of the five-pulse experiment, however their intensity under the present experimental conditions was comparable to the noise level.

The interfering echoes can be removed by phase cycling. Different phase cycles involving both observer and pump pulse phase cycling were again generated and analysed (see Table 2). The eight-step phase cycle $(x x) x_{\mathrm{p}}[x] x_{\mathrm{p}}$ involving only the observer pulses removes all of the crossing echoes except for the following refocused echoes: $\mathrm{PE}_{1 \mathrm{p}} \mathrm{r}_{3}$ crossing at $t_{\mathrm{DEER}}=$ $\tau_{1}+\tau_{2}-t_{2}, \mathrm{PE}_{2 \mathrm{p}} \mathrm{r}_{3}$ crossing at $t_{\mathrm{DEER}}=\frac{1}{2} \tau_{1}+\tau_{2}-t_{2}, \mathrm{PE}_{12} \mathrm{r}_{\mathrm{p}} \mathrm{r}_{3}$ crossing at $t_{\text {DEER }}=\tau_{2}-t_{2}, \mathrm{PE}_{1 \mathrm{p}} \mathrm{r}_{3} \mathrm{r}_{\mathrm{p}^{\prime}}$ crossing at $t_{\mathrm{DEER}}=\tau_{1}$, $\mathrm{PE}_{2 \mathrm{p}} \mathrm{r}_{3} \mathrm{r}_{\mathrm{p}^{\prime}}$ crossing at $t_{\mathrm{DEER}}=\frac{1}{2} \tau_{1}$ and $\mathrm{PE}_{12} \mathrm{r}_{\mathrm{p}} \mathrm{r}_{3} \mathrm{r}_{\mathrm{p}^{\prime}}$ crossing at $t_{\text {DEER }}=0$. However, these echoes are only formed for spin packets refocused by both observer and pump pulses and therefore have negligible intensity in the absence of significant excitation bandwidth overlap. This phase cycle also removes all of the standing echoes close to the detection window, leaving only the primary echo $\mathrm{PE}_{23}$ separated by $\tau_{1}$ from the detected refocused echo. The five-pulse DEER trace recorded with this phase cycle is also shown in the top panel of Fig. 8A, demonstrating that this phase cycle efficiently removes the distortions introduced by the crossing echoes.

Considering only observer pulse phase cycling, a 64-step phase cycle, cycling each of the observer pulses in steps of $90^{\circ}$, would be required to remove most of the remaining crossing echoes, but does not remove the contribution of the triply refocused echo $\mathrm{PE}_{12} \mathrm{r}_{\mathrm{p}} \mathrm{r}_{3} \mathrm{r}_{\mathrm{p}^{\prime}}$ at $t_{\mathrm{DEER}}=0$. On the other hand, $\mathrm{a}$ 16-step phase cycle involving both observer and pump pulses, $x x\left[x_{\mathrm{p}}\right][x] x_{\mathrm{p}}$ (Table 2), removes all of the crossing echoes and the standing echoes close to the detection window (except $\mathrm{PE}_{23}$ as above). The same phase cycles can also be used with the starting phase of the refocusing observer pulses set to $+y$ (i.e. $(x y) x_{\mathrm{p}}[y] x_{\mathrm{p}}$ instead of $(x x) x_{\mathrm{p}}[x] x_{\mathrm{p}},[+(+x,+y)-(-x,-y)]$ phase cycle for the first two observer pulses and $[+(+y)-(-x)+(-y)$ $-(+x)]$ phase cycle for the last observer pulse), as typically used in Carr-Purcell-Meiboom-Gill refocusing sequences. ${ }^{34}$

3.1.2 Forward five-pulse DEER. Similar considerations apply to the forward five-pulse DEER experiment. The corresponding data is shown in the ESI† (Fig. S13). Again, the main distortions are caused by similar types of echoes, in particular, the primary echo of the two pump pulses $\mathrm{PE}_{\mathrm{p}^{\prime} \mathrm{p}}$, the refocused primary echo $\mathrm{PE}_{1 \mathrm{p}^{\prime}} \mathrm{r}_{\mathrm{p}}$, the primary echo $\mathrm{PE}_{2 \mathrm{p}}$ and the stimulated echoes $\mathrm{SE}_{\mathrm{p}^{\prime} \mathrm{p} 3}$ and $\mathrm{SE}_{2 \mathrm{p} 3}$. The most significant distortions are similarly caused by the primary echo $\mathrm{PE}_{\mathrm{p}^{\prime} \mathrm{p}}$, but are less severe as this echo crosses the detection window for a longer delay between the two pump pulses compared to reverse five-pulse DEER and has thus decayed more significantly due to relaxation. In addition to this, the crossing point occurs at a later point in the DEER trace $\left(t_{\text {DEER }}=\frac{1}{2} \tau_{1}+\tau_{2}-\frac{3}{2} t_{2}\right)$, where most of the modulations will likely already have decayed.

The observed distortions can again be removed by phase cycling (Table 2). The eight-step phase cycle $x x_{\mathrm{p}}[x] x_{\mathrm{p}}(x)$ involving only the observer pulses removes all crossing echoes except for echoes refocused by both observer and pump pulses and therefore exhibiting negligible intensity under the present experimental conditions. Specifically, the echoes not removed by this phase cycle are: $\mathrm{PE}_{12} \mathrm{r}_{\mathrm{p}}$ crossing at $t_{\mathrm{DEER}}=\tau_{1}+\tau_{2}-t_{2}$, $\mathrm{PE}_{\mathrm{p}^{\prime} 2} \mathrm{r}_{\mathrm{p}}$ crossing at $t_{\mathrm{DEER}}=\frac{1}{2} \tau_{1}+\tau_{2}-\frac{1}{2} t_{2}, \mathrm{PE}_{1 \mathrm{p}^{\prime}} \mathrm{r}_{2} \mathrm{r}_{\mathrm{p}}$ crossing at $t_{\mathrm{DEER}}=\tau_{2}, \mathrm{PE}_{12} \mathrm{r}_{\mathrm{p}} \mathrm{r}_{3}$ crossing at $t_{\mathrm{DEER}}=\tau_{1}-t_{2}, \mathrm{PE}_{\mathrm{p}^{\prime} 2} \mathrm{r}_{\mathrm{p}} \mathrm{r}_{3}$ crossing at $t_{\text {DEER }}=\frac{1}{2} \tau_{1}-\frac{1}{2} t_{2}$ and $\mathrm{PE}_{1 \mathrm{p}^{\prime}} \mathrm{r}_{2} \mathrm{r}_{\mathrm{p}} \mathrm{r}_{3}$ crossing at $t_{\mathrm{DEER}}=0$. The only remaining standing echoes that approach the detection window in experiments with certain inter-pulse delays are the refocused primary echoes $\mathrm{PE}_{\mathrm{p}^{\prime} 2} \mathrm{r}_{3}$ and $\mathrm{PE}_{1 \mathrm{p}^{\prime}} \mathrm{r}_{2} \mathrm{r}_{3}$, separated by $\tau_{1}-t_{2}$ and $2 \tau_{1}-2 t_{2}$ from the detected refocused echo. Again, a 64-step phase cycle on the observer pulses would be required to remove all crossing echoes, and would still leave the contribution of the triply refocused echo $\mathrm{PE}_{1 \mathrm{p}^{\prime}} \mathrm{r}_{2} \mathrm{r}_{\mathrm{p}} \mathrm{r}_{3}$ at $t_{\mathrm{DEER}}=0$. A 16-step phase cycle, analogous to the one proposed for reverse fivepulse DEER, $x x_{\mathrm{p}}[x]\left[x_{\mathrm{p}}\right] x$, removes all of the crossing echoes and the standing echoes close to the detection window (except for the two refocused primary echoes mentioned above).

\subsection{Seven-pulse DEER}

In the seven-pulse DEER experiment, the dipolar evolution time is extended even further by adding another refocusing observer pulse and an additional pump pulse to the five-pulse DEER experiment (see Fig. 1). ${ }^{14,21}$ Similarly to the five-pulse experiment, $\tau_{1}, \tau_{2}$ and $\tau_{3}$ are chosen to be equal or almost equal to maximize echo intensity. The time axis of the seven-pulse DEER experiment is given by $t_{\mathrm{DEER}}=\tau_{1}+\tau_{2}+\tau_{3}-t_{1}-t_{2}-t_{3}$ for delays as indicated in Fig. 1. The maximum dipolar evolution time for this sequence corresponds to $\tau_{1}+\tau_{2}+\tau_{3}-t_{1,0}-t_{2}-t_{3,0}$, where $t_{1,0}$ and $t_{3,0}$ refer to the initial values for the corresponding delays. 
The seven pulses give $3^{N-1}=729$ coherence transfer pathways starting from thermal equilibrium and ending with observer spin coherence order -1 . A total of 665 echoes can potentially be formed and more than 200 of these cross the detection window. In addition to the crossing echoes, several standing echoes partially or completely overlap with the desired refocused echo $\mathrm{PE}_{12} \mathrm{r}_{3} \mathrm{r}_{4}$ at $2\left(\tau_{1}+\tau_{2}+\tau_{3}\right)$ and have to be removed by phase cycling.

The positions of the echoes during a seven-pulse DEER experiment performed with inter-pulse delays $\tau_{1}=1.9 \mu \mathrm{s}$, $\tau_{2}=2.1 \mu \mathrm{s}$ and $\tau_{3}=2.2 \mu \mathrm{s}$ are shown in Fig. 9A along with the corresponding DEER trace recorded without phase cycling. The apparent oscillation with a maximum at about $2 \mu \mathrm{s}$ is due to the contribution of the additional population transfer pathways. Distortions due to crossing echoes can clearly be identified around $1.7 \mu$ s and in the range from about $3.5 \mu$ s to $5.0 \mu \mathrm{s}$. Oscillations in the quadrature part of the signal indicate the presence of additional artefacts around time zero and in the initial part of the trace, where artefacts can introduce large errors in distance distributions determined by Tikhonov regularization. Echo transients recorded at different times during the DEER experiment show several relatively strong echoes induced by the coherent pump pulses (see Fig. 9B). The primary echoes $\mathrm{PE}_{\mathrm{pp}^{\prime \prime \prime}}$ and $\mathrm{PE}_{\mathrm{p}^{\prime \prime} \mathrm{p}^{\prime \prime \prime}}$ formed by pairs of pump pulses, could be clearly identified and cross the detected echo at around $t_{\mathrm{DEER}}=\frac{1}{3}\left(\tau_{1}-\tau_{2}\right)+\tau_{3}-t_{2}$ and $t_{\mathrm{DEER}}=\tau_{1}+\tau_{3}$. Due to the opposite frequency sweep direction of the pump pulse $\mathrm{p}^{\prime \prime}$, the latter echo is much broader and introduces distortions in a larger region of the DEER trace. Other echoes are also characterized by non-negligible intensities but could not be unequivocally identified due to their large number and broad nature.

A series of phase cycles capable of removing the observed distortions were considered (see Table 2). The 32-step phase cycle $x[x] x_{\mathrm{p}}(x)\left(x_{\mathrm{p}}\right)\left(x_{\mathrm{p}}\right) x$ removes the standing echoes close to the detection window and most of the crossing echoes, leaving only nine primary echoes refocused by both observer and pump pulses, eight of which cross around $t_{\text {DEER }}=\tau_{1}$ for similar $\tau_{1}, \tau_{2}$ and $\tau_{3}$ values and the other at $t_{\text {DEER }}=0$ (see ESI, $\dagger$ Section 4.2 for more information). The DEER trace recorded using this phase cycle is shown in the top panel of Fig. 9A. All of the echo crossing artefacts visible in the trace recorded without phase cycling are removed. The trace recorded with only the observer part of this phase cycle, the eight-step phase cycle $x[x] x_{\mathrm{p}}(x) x_{\mathrm{p}} x_{\mathrm{p}} x$, is shown for comparison and still contains the artefact at about $1.7 \mu \mathrm{s}$. This phase cycle is therefore not sufficient. All of the moving echoes crossing the detection window during a seven-pulse DEER experiment with $0 \leq t_{\text {DEER }} \leq$ $\tau_{1}+\tau_{2}+\tau_{3}-t_{1,0}-t_{2}-t_{3,0}$ are removed in the 128-step phase cycle $[x x]\left[x_{\mathrm{p}}\right] x\left[x_{\mathrm{p}} x_{\mathrm{p}}\right](x)$. A 256-step phase cycle, $x x\left[x_{\mathrm{p}}\right][x] x_{\mathrm{p}}\left[x_{\mathrm{p}}\right][x]$, would be required to remove all of the moving echoes. Amongst the phase cycles only involving the observer pulses, the 128-step phase cycle $[x][x] x_{\mathrm{p}}[x] x_{\mathrm{p}} x_{\mathrm{p}}(x)$ only leaves two crossing echoes, and the full 256-step phase cycle $[x][x] x_{\mathrm{p}}[x] x_{\mathrm{p}} x_{\mathrm{p}}[x]$ removes all of the crossing echoes.

In summary, in the multi-pulse DEER experiments, the most significant distortions are caused by primary echoes formed by two pump pulses, which are characterized by intensities

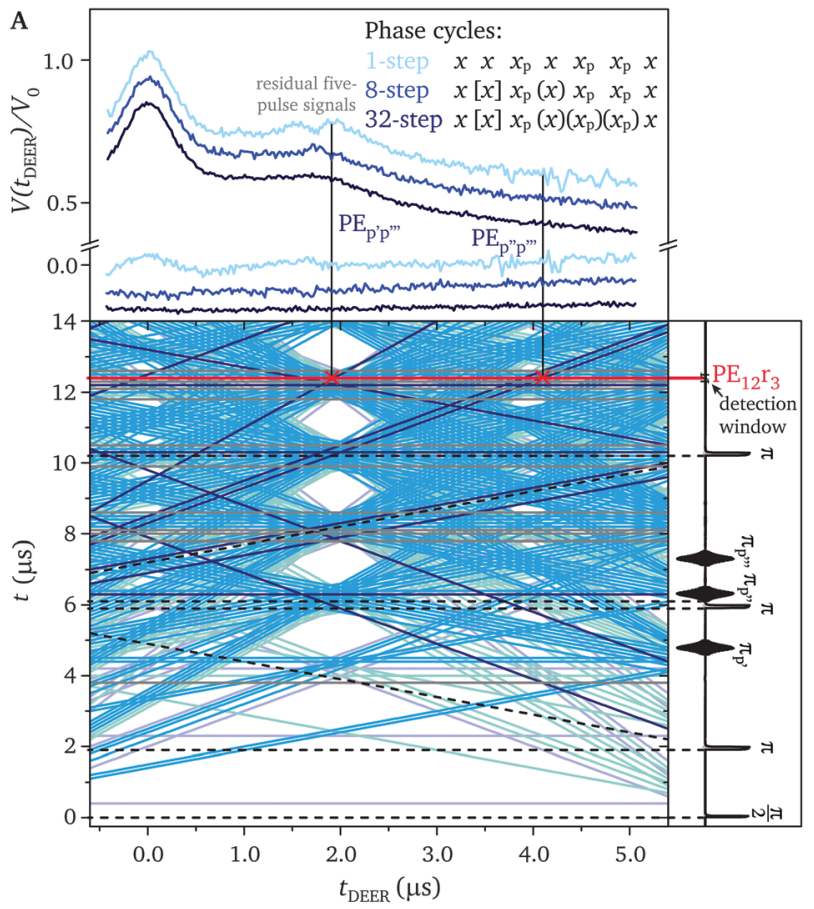

B

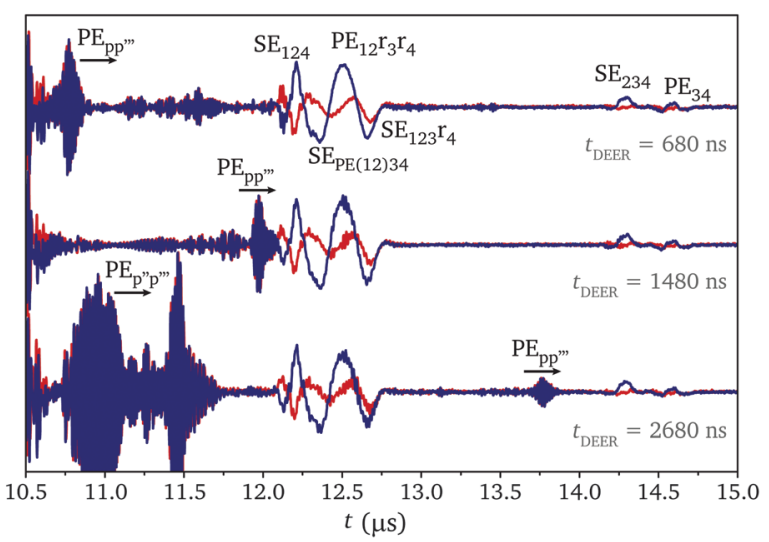

Fig. 9 (A) Positions of the echo crossings in a seven-pulse DEER experiment with the following inter-pulse delays $\tau_{1}=1.9 \mu \mathrm{s}, \tau_{2}=2.1 \mu \mathrm{s}, \tau_{3}=2.2 \mu \mathrm{s}$ and $t_{2}=200 \mathrm{~ns}$. The pulse sequence is plotted along the $y$-axis with pulse positions represented by dashed lines. The standing echoes of the observer sequence are shown in grey and the different types of echoes induced by the pump pulses are shown in different shades of blue following the same colour code as in Fig. 3. Seven-pulse DEER traces recorded with different phase cycles are shown in the top panel. The traces are offset to allow better comparison. The crossing positions of the two strongest moving echoes with the detected refocused echo $\left(\mathrm{PE}_{12} \mathrm{r}_{3}\right.$, position shown in red) are highlighted and tentatively assigned. (B) Echo transients recorded at three points in the DEER experiment. The echoes that could be assigned are labelled following the conventions used for the four- and five-pulse experiments and the direction of movement for crossing echoes is indicated by arrows.

comparable to those of the detected refocused echo. These distortions were found to be most severe for the reverse fivepulse DEER experiment. In case of both versions of the fivepulse DEER experiment, however, an eight-step phase cycle, or a 16-step phase cycle including pump pulse phase cycling in 
case of increased overlap of the pulse excitation profiles, efficiently removes the corresponding echo crossing artefacts. In the seven-pulse DEER experiment, a 32-step phase cycle involving changes in the phases of both observer and pump pulses was found to be required to remove echo crossing artefacts in the absence of significant excitation bandwidth overlap. The proposed phase cycling schemes therefore enable the use of the five- and seven-pulse DEER experiments for the measurement of long inter-spin distances in the presence of a coherent pump channel.

\section{Conclusions}

The recent advances in technology allowing the use of pulse shaping in EPR and leading to the concept of fully coherent AWG-based spectrometers open up a range of new possibilities through an increased level of control over coherent spin manipulations. However, this also introduces new aspects to be considered in the design and application of advanced pulse sequences. In this paper, we have analysed the effects of coherent observer and pump pulses in the standard and in multi-pumppulse DEER experiments. We have shown that significant distortions in the DEER trace occur due to echoes induced by pulses at both frequencies crossing the detection window.

The shapes and intensities of the echoes formed by coherence transfer pathways involving both observer and pump pulses were demonstrated to be determined mainly by the length of the pulses and the overlap of their excitation profiles. Even in the absence of significant excitation profile overlap, the off-resonant effect of the pulses is sufficient to generate primary and stimulated echoes of appreciable intensity. The intensity of refocused echoes, due to pathways involving coherence transfer induced by pulses at two different frequencies, on the other hand, were shown to be negligible under these conditions. These results highlight the importance of considering off-resonant effects in multi-frequency experiments, both in the case of coherent pulses at fixed frequencies or in the presence of frequency-swept pulses.

The crossing points of the different additional echoes in four-, five- and seven-pulse DEER experiments with coherent pump pulses were determined and the corresponding artefacts identified in the DEER traces. The introduced distortions can be severe under typical experimental conditions and could in some cases be difficult to clearly distinguish from dipolar modulations, hence they need to be removed to avoid misinterpretation. This was shown to be possible through phase cycling. The negligible intensity of echoes refocused by pulses at different frequencies allows the use of phase cycles with fewer steps compared to phase cycles that would be required to suppress all of the coherence transfer pathways leading to echo crossings within the dipolar evolution window. The use of both observer and pump pulse phase cycling also reduces the number of required phase cycling steps. Eight-step phase cycles were shown to be sufficient to suppress echo crossing artefacts in four- and five-pulse DEER and at least a 32-step phase cycle was shown to be required for seven-pulse DEER. The undesired effects of coherent pump pulses in the standard four-pulse and the novel multi-pump-pulse DEER experiments performed on nitroxide-labelled proteins can therefore be efficiently eliminated through appropriate phase cycling, enabling the use of these pulse sequences on fully coherent AWG-based spectrometers.

The excellent agreement between experimental echo transients and simulations based on a density matrix approach demonstrates that all of the signals, including those due to off-resonant effects, can be quantitatively simulated using a simple theoretical approach. This approach can be extended to the description of other multi-frequency experiments on fully coherent EPR spectrometers. The inclusion of the instrument transfer functions for both the excitation and detection paths of the spectrometer was found to be crucial for an accurate theoretical description of the spin response. The ability to accurately model the spin response under specific instrumental conditions is at the basis of further improvements in sensitivity and resolution of pulse EPR experiments through optimization of analytical pulse shapes and the design of optimal control pulses. ${ }^{13,17}$

\section{Materials and methods}

\subsection{Instrumentation}

A Bruker Elexsys E580 X/Q-band spectrometer extended by a custom-made pulse shaping channel, similar to that described in ref. 13, was used for the pulse EPR measurements (see Fig. 10). Shaped pulses were generated by a Keysight $81180 \mathrm{~B}$ AWG (two channels, $4.6 \mathrm{GSa} \mathrm{s}^{-1}, 1 \mathrm{GHz}$ analog bandwidth, 12 bit vertical resolution) independently controlled by a separate computer. The pulses are programmed into the two channels of the AWG and upconverted to X-band frequencies using an IQ mixer (Marki MLIQ-0416, LO/RF 4-16 GHz, IF DC-3.5 GHz, LO drive level 11-18 dBm, $-45 \mathrm{~dB}$ LO-RF isolation at $9 \mathrm{GHz}$ ) driven by an LO derived from the microwave source of the E580 bridge and amplified to about $15 \mathrm{dBm}$ (with a Miteq AFSD5-080120-25-26P amplifier). Isolators (Meuro CIS080120F) protect the bridge from reflections. The pulses from the AWG channel are therefore coherent with the pulses on the internal pulse forming channels (SPFU, Stripline Pulse Forming Unit) of the spectrometer. The additional pulse channel was installed in parallel to the internal SPFU channels and follows the same signal routing

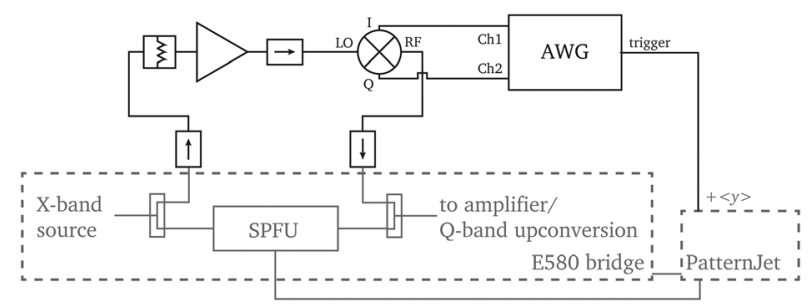

Fig. 10 Schematic block diagram of the homebuilt AWG extension to the Bruker Elexsys X/Q-band spectrometer. The X-band microwave signal from the power splitter before the spectrometer's pulse forming channels is amplified and drives an IQ mixer connected to the two channels of the AWG. The pulses from the AWG are routed back into the bridge through the power combiner after the pulse forming channels. 
pathway to the resonator. The AWG channel is triggered from the $+\langle y\rangle$ channel of the spectrometer, which also controls the corresponding pulse gate and receiver protection. For Q-band measurements, the pulses are upconverted and amplified in a Bruker SuperQ-FT bridge equipped with a $3 \mathrm{~W}$ solid-state amplifier. A calibration is performed to minimize the phase and amplitude imbalance of the I and Q channels and to minimize LO leakage (contribution of the spectrometer frequency $\nu_{\mathrm{obs}}$ to the AWG signals) by applying a DC offset to the I and Q channels of the AWG following a procedure similar to the one reported in ref. 24 .

The measurements were performed with an overcoupled dielectric resonator (Bruker EN5107D2, Q-factor of ca. 300-400) at $50 \mathrm{~K}$ using a cryogen-free cooling system (Bruker ER 4118HV-CF58).

In order to accurately model the experimental echo signals, knowledge of the transfer functions of the instrumental excitation and detection paths is required. The spectrometer transfer function was therefore determined following the procedure described in ref. 15. The resulting transfer function can be used to derive the resonator impulse response affecting both excitation and signal detection. Further details can be found in the ESI. $\dagger$ The internal video amplifier in the detection pathway of the E580 spectrometer is also characterized by a non-flat frequency response in the frequency region of interest ${ }^{13}$ and needs to be taken into account in the simulations. The frequency response was measured by recording the output amplitude of rectangular pulses programmed in the AWG and directly input to the video amplifier as a function of frequency and video gain. Further details and plots of the experimental frequency response functions can be found in the ESI. $\dagger$ The video amplifier bandwidth was set to $200 \mathrm{MHz}$ for all the measurements reported here.

\subsection{EPR measurements}

The EPR measurements were performed on complexes of the HCN2 (hyperpolarisation-activated cyclic nucleotide-gated ion channel) protein doubly spin-labelled at residues $\mathrm{S} 563 \mathrm{C}$ and R635C (four-pulse DEER measurements) or residues V537C and R635C (five- and seven-pulse DEER measurements) with Trip $8 \mathrm{~b}_{\text {core }}$ (truncated tetratricopeptide repeat-containing Rab8b-interacting protein, residues 223-303). The EPR samples were prepared as described in ref. 35.

Four-pulse DEER data were recorded with the pulse sequence $\frac{\pi}{2}{ }_{\text {obs }}-\tau_{1}-\pi_{\text {obs }}-t_{1}-\pi_{\text {pump }}-\left(\tau_{1}+\tau_{2}-t_{1}\right)-\pi_{\text {obs }}-\tau_{2}$ (see Fig. 1) with observer pulse lengths $t_{\pi / 2}=32 \mathrm{~ns}$ and $t_{\pi}=64 \mathrm{~ns}$ and delay times $\tau_{1}=400 \mathrm{~ns}$ and $\tau_{2}=2.3 \mu \mathrm{s}$. The pump pulse position was varied in $10 \mu \mathrm{s}$ increments. The time axis of the experiment is $t_{\text {DEER }}=t_{1}-\tau_{1}$. The pump pulse was either a 64 ns monochromatic rectangular pulse at a frequency of $\nu_{\text {pump }}=\nu_{\text {obs }}+70 \mathrm{MHz}$ or a $200 \mathrm{~ns}$ sech/tanh pulse centred at $\nu_{\text {pump }}=\nu_{\mathrm{obs}}+75 \mathrm{MHz}$ and with the following amplitude and frequency modulation functions: ${ }^{16}$

$$
\begin{gathered}
B_{1}(t)=B_{1}^{\max } \operatorname{sech}(\beta t) \\
\nu(t)=\frac{1}{2} \mathrm{BW}_{\infty} \tanh (\beta t)
\end{gathered}
$$

where $B_{1}^{\max }$ is the maximum $B_{1}$ field, $\beta$ is a truncation parameter, $\mathrm{BW}_{\infty}$ is the pulse bandwidth for $\tanh (\beta t)$ (the pulse sweeps over the range $\left.\pm \mathrm{BW}_{\infty} / 2\right)$ and $t$ is defined in the range from $-t_{\mathrm{p}} / 2$ to $+t_{\mathrm{p}} / 2$. The phase modulation function $\phi(t)$ is obtained as the integral of the frequency modulation function

$$
\phi(t)=\frac{1}{2} \frac{\mathrm{BW}_{\infty}}{\beta} \log [\cosh (\beta t)]
$$

and the in-phase (I) and quadrature (Q) parts of the sech/tanh pulse are computed as the real and imaginary parts of:

$$
y(t)=B_{1}(t) \mathrm{e}^{2 \pi i[\phi(t)+\Delta \nu]}
$$

where $\Delta \nu$ is the offset with respect to the observer frequency.

The parameters $\beta$ and $\mathrm{BW}_{\infty}$ were set to $4 / t_{\mathrm{p}}$ and $70 \mathrm{MHz}$ (i.e. the sech/tanh pulse sweeps over the range $\nu_{\text {pump }}=\nu_{\text {obs }}+$ $75 \mathrm{MHz} \pm 35 \mathrm{MHz}$ ), respectively. These pulse parameters were determined using the optimization method described in ref. 20 (see ESI $\dagger$ for details). For both monochromatic and sech/ tanh pump pulses, the central pump frequency approximately matched the maximum of the nitroxide spectrum and was set to about $20 \mathrm{MHz}$ above the frequency of the centre of the resonator dip. Reference experiments were performed with a $64 \mathrm{~ns}$ monochromatic rectangular pump pulse from the incoherent ELDOR channel of the Bruker spectrometer.

Five-pulse DEER data were recorded with the pulse sequence $\overline{2}_{\text {obs }}-\left(\tau_{1}-t_{2}\right)-\pi_{\text {pump }}-t_{2}-\pi_{\text {obs }}-t_{1}-\pi_{\text {pump }}-\left(\tau_{1}+\tau_{2}-t_{1}\right)-$ $\pi_{\mathrm{obs}}-\tau_{2}$ for the forward version of the experiment (second pump pulse before the second observer pulse) or with the sequence $\frac{\pi}{2}{ }_{\text {obs }}-\tau_{1}-\pi_{\text {obs }}-\left(\tau_{1}+\tau_{2}-t_{1}\right)-\pi_{\text {pump }}-t_{1}-\pi_{\text {obs }}-$ $t_{2}-\pi_{\text {pump }}-\left(\tau_{2}-t_{2}\right)$ for the reverse version of the experiment (second pump pulse after the last observer pulse) (see Fig. 1). In both cases, the maximum of the DEER trace (zero-time) occurs at $t_{1}=t_{2}$ and the time axis of the experiment corresponds to $t_{\text {DEER }}=t_{1}-t_{2}$, where $t_{1}$ is the delay as indicated in Fig. 1 . The five-pulse DEER experiments were performed with observer pulse lengths $t_{\pi / 2}=40 \mathrm{~ns}$ and $t_{\pi}=80 \mathrm{~ns}$ and delay times $\tau_{1}=2.1 \mu \mathrm{s}$ and $\tau_{2}=2.4 \mu \mathrm{s}$. In the forward five-pulse DEER experiment, the delay $t_{2}$, defined as the delay between the start of the pulses, was set to $800 \mathrm{~ns}$, and in the reverse five-pulse DEER experiment $t_{2}$ was set to $400 \mathrm{~ns}$. The initial delay between the second observer pulse and the moving pump pulse was set to $100 \mathrm{~ns}$ in both cases. The position of the pump pulse during the delay $\tau_{1}+\tau_{2}$ was varied in $10 \mathrm{~ns}$ increments.

Seven-pulse DEER experiments were performed with the pulse sequence $\frac{\pi}{2}{ }_{\mathrm{obs}}-\tau_{1}-\pi_{\mathrm{obs}}-t_{1}-\pi_{\mathrm{pump}}-\left(\tau_{1}+\tau_{2}-t_{1}\right)-\pi_{\mathrm{obs}}-t_{2}-$ $\pi_{\text {pump }}-\left(\tau_{2}+\tau_{3}-t_{2}-t_{3}\right)-\pi_{\text {pump }}-t_{3}-\pi_{\text {obs }}-\tau_{3}$ (see Fig. 1). The seven-pulse DEER experiments were performed with observer pulse lengths $t_{\pi / 2}=40 \mathrm{~ns}$ and $t_{\pi}=80 \mathrm{~ns}$ and delay times $\tau_{1}=1.9 \mu \mathrm{s}, \tau_{2}=2.1 \mu \mathrm{s}$ and $\tau_{3}=2.2 \mu \mathrm{s}$. The initial pump pulse delays were set to $t_{1,0}=t_{2}=t_{3,0}=200 \mathrm{~ns}$. The position of the first pump pulse $\left(t_{1}\right)$ was incremented in $10 \mathrm{~ns}$ steps and the position of the third pump pulse was simultaneously decremented in $10 \mathrm{~ns}$ steps ( $t_{3}$ incremented). The experiment time axis is obtained as $t_{\text {DEER }}=\tau_{1}+\tau_{2}+\tau_{3}-t_{1}-t_{2}-t_{3}$ (with delays as labelled in Fig. 1) and therefore $\Delta t=20 \mathrm{~ns}$. The maximum of the DEER trace (zero-time) occurs when $t_{1}=t_{3}=\frac{1}{2}\left(\tau_{1}+\tau_{2}+\tau_{3}-t_{2}\right)$. 
The pump pulses in the five-pulse and seven-pulse DEER experiments were $400 \mathrm{~ns}$ sech/tanh pulses with the parameters $\beta$ and $\mathrm{BW}_{\infty}$ set to $6 / t_{\mathrm{p}}$ and $35 \mathrm{MHz}$, respectively, and centred at $\nu_{\text {pump }}=\nu_{\text {obs }}+60 \mathrm{MHz}$. In this case, the pump frequency was set to the centre frequency of the resonator dip. The pulse length was increased and the bandwidth decreased compared to the pump pulse in the four-pulse experiment in order to maximize the inversion efficiency and optimize the inversion profile for the limited available power. In DEER experiments with multiple pump pulses, a high uniform inversion efficiency is required to reduce the contribution of signals from population transfer pathways determined by spins affected only by a subset of the pump pulses. ${ }^{14,21}$ In the four-pulse DEER experiment, on the other hand, a reduced inversion efficiency over a larger bandwidth can still lead to a more significant increase in modulation depth. The pump pulse optimization was performed by measurement of the experimental inversion profiles as a function of pulse length, bandwidth and $\beta$ parameter and using the method described in ref. 20 to optimize the frequency offset (see ESI $\dagger$ for details). The frequency sweep direction of one of the pump pulses was reversed by inverting the sign of the quadrature part of the sech/tanh pulse to achieve an offsetindependent net dipolar evolution time in the five-pulse DEER experiment and to reduce the extent of the distribution of dipolar evolution times for spins at different frequency offsets in the seven-pulse DEER experiment. ${ }^{16}$

The shot repetition time was set to $5 \mathrm{~ms}$ for all experiments. Phase cycling was performed as described in the main text.

Two- and three-pulse echo transients were recorded for different combinations of $\frac{\pi}{2}$ and $\pi$ pulses at the observer and pump frequencies. The pulse parameters were the same as described above for the four-pulse DEER experiment. An interpulse delay of $600 \mathrm{~ns}$ was used for the two-pulse sequence and inter-pulse delays of $600 \mathrm{~ns}$ and $1800 \mathrm{~ns}$ were used for the threepulse sequence. For the echo measurements, the observer frequency was set to $33.860 \mathrm{GHz}$ and the magnetic field was set to $1208.9 \mathrm{mT}$ corresponding to the nitroxide maximum at the pump frequency $(\Delta \nu=+70 \mathrm{MHz})$. A total of $2^{16}$ transients were averaged for the two-pulse echoes and averaging over $2^{17}$ transients was performed for the three-pulse echoes.

\subsection{Simulations}

The echo simulations were performed with a custom Matlab program implementing the spin density matrix evolution for a two-level system in Hilbert space. The simulations were performed in a frame rotating at the observer frequency. The effect of offresonant and shaped pulses was simulated by step-wise propagation using a time step of $1 \mathrm{~ns} .{ }^{36}$ Spin relaxation was neglected.

The effect of the limited bandwidth of the resonator on the transmitted pulses and the returned echo signal was included by convolution of both the pulse shapes and the calculated echo signals with the resonator impulse response. The impulse response function was derived as described in ref. 15 and 36 based on the frequency response of the resonator measured through nutation experiments (see Section 5.1 and ESI†).
The effect of the video amplifier on the echo signals was modelled by a digital filter based on the measured frequency response of the video amplifier (see Section 5.1 and ESI $\dagger$ ) at the experimental video gain setting.

\section{Acknowledgements}

This work was supported by NIH grant R01EY010329, NSF grant CHE-1452967 and American Heart Association grant 14CSA20380095.

\section{References}

1 Structural Information from Spin-Labels and Intrinsic Paramagnetic Centres in the Biosciences, Structure and Bonding 152, ed. C. R. Timmel and J. Harmer, Springer Verlag, Berlin, Heidelberg, 2014.

2 Distance Measurements in Biological Systems by EPR, Biological Magnetic Resonance, ed. L. J. Berliner, S. S. Eaton and G. R. Eaton, Kluwer Academic/Plenum Publishers, New York, 2000, vol. 19.

3 B. Joseph, V. M. Korkhov, M. Yulikov, G. Jeschke and E. Bordignon, J. Biol. Chem., 2014, 289, 3176-3185.

4 M. T. Lerch, Z. Yang, E. K. Brooks and W. L. Hubbell, Proc. Natl. Acad. Sci. U. S. A., 2014, 111, E1201-E1210.

5 B. Joseph, A. Sikora, E. Bordignon, G. Jeschke, D. S. Cafiso and T. F. Prisner, Angew. Chem., Int. Ed., 2015, 54, 6196-6199.

6 V. Meyer, M. A. Swanson, L. J. Clouston, P. J. Boratyński, R. A. Stein, H. S. McHaourab, A. Rajca, S. S. Eaton and G. R. Eaton, Biophys. J., 2015, 108, 1213-1219.

7 D. Constantinescu-Aruxandei, B. Petrovic-Stojanovska, O. Schiemann, J. H. Naismith and M. F. White, Nucleic Acids Res., 2016, 44, 954-968.

8 G. Jeschke and Y. Polyhach, Phys. Chem. Chem. Phys., 2007, 9, 1895-1910.

9 G. Jeschke, V. Chechik, P. Ionita, A. Godt, H. Zimmermann, J. E. Banham, C. R. Timmel, D. Hilger and H. Jung, Appl. Magn. Reson., 2006, 30, 473-498.

10 M. K. Bowman and A. G. Maryasov, J. Magn. Reson., 2007, 185, 270-282.

11 V. V. Kurshev, A. M. Raitsimring and Y. D. Tsvetkov, J. Magn. Reson., 1989, 81, 441-454.

12 G. Jeschke, Structural Information from Spin-Labels and Intrinsic Paramagnetic Centres in the Biosciences, Structure and Bonding 152, ed. C. R. Timmel and J. Harmer, Springer Verlag, Berlin, Heidelberg, 2014, ch. 2, pp. 83-120.

13 P. E. Spindler, Y. Zhang, B. Endeward, N. Gershernzon, T. E. Skinner, S. J. Glaser and T. F. Prisner, J. Magn. Reson., 2012, 218, 49-58.

14 P. P. Borbat, E. R. Georgieva and J. H. Freed, J. Phys. Chem. Lett., 2013, 4, 170-175.

15 A. Doll, S. Pribitzer, R. Tschaggelar and G. Jeschke, J. Magn. Reson., 2013, 230, 27-39.

16 P. E. Spindler, S. J. Glaser, T. E. Skinner and T. F. Prisner, Angew. Chem., Int. Ed., 2013, 52, 3425-3429. 
17 A. Doll and G. Jeschke, J. Magn. Reson., 2014, 246, 18-26.

18 G. Jeschke, S. Pribitzer and A. Doll, J. Phys. Chem. B, 2015, 119, 13570-13582.

19 A. Doll, M. Qi, S. Pribitzer, N. Wili, M. Yulikov, A. Godt and G. Jeschke, Phys. Chem. Chem. Phys., 2015, 17, 7334-7344.

20 A. Doll, M. Qi, N. Wili, S. Pribitzer, A. Godt and G. Jeschke, J. Magn. Reson., 2015, 259, 153-162.

21 P. E. Spindler, I. Waclawska, B. Endeward, J. Plackmeyer, C. M. Ziegler and T. F. Prisner, J. Phys. Chem. Lett., 2015, 6, 4331-4335.

22 T. F. Segawa, A. Doll, S. Pribitzer and G. Jeschke, J. Chem. Phys., 2015, 143, 044201.

23 P. Schöps, P. E. Spindler, A. Marko and T. F. Prisner, J. Magn. Reson., 2015, 250, 55-62.

24 T. Kaufmann, T. J. Keller, J. M. Franck, R. P. Barnes, S. J. Glaser, J. M. Martinis and S. Han, J. Magn. Reson., 2013, 235, 95-108.

25 G. Bodenhausen, H. Kogler and R. R. Ernst, J. Magn. Reson., 1984, 58, 370-388.
26 C. Gemperle, G. Aebli, A. Schweiger and R. R. Ernst, J. Magn. Reson., 1990, 88, 241-256.

27 S. Stoll and B. Kasumaj, Appl. Magn. Reson., 2008, 35, 15-32.

28 A. Schweiger and G. Jeschke, Principles of pulse electron paramagnetic resonance, Oxford University Press, 2001.

29 E. Kupče and R. Freeman, J. Magn. Reson., Ser. A, 1995, 117, 246-256.

30 F. Bloch and A. Siegert, Phys. Rev., 1940, 57, 522-527.

31 N. F. Ramsey, Phys. Rev., 1955, 100, 1191-1194.

32 L. Emsley and G. Bodenhausen, Chem. Phys. Lett., 1990, 168, 297-303.

33 D. I. Hoult and R. E. Richards, Proc. R. Soc. London, Ser. A, 1975, 344, 311-340.

34 S. Meiboom and D. Gill, Rev. Sci. Instrum., 1958, 29, 688-691.

35 H. A. DeBerg, J. R. Bankston, J. C. Rosenbaum, P. S. Brzovic, W. N. Zagotta and S. Stoll, Structure, 2015, 23, 734-744.

36 S. Pribitzer, A. Doll and G. Jeschke, J. Magn. Reson., 2016, 263, 45-54. 